\title{
Approximation of Time-Dependent, Multi-Component, Viscoelastic Fluid Flow
}

\author{
Vincent J. Ervin*¥ William W. Miles ${ }^{\dagger \ddagger}$
}

\begin{abstract}
In this article we analyse a fully discrete approximation to the time dependent viscoelasticity equations allowing for multicomponent fluid flow. The Oldroyd B constitutive equation is used to model the viscoelastic stress. For the discretization, time derivatives are replaced by backward difference quotients, and the non-linear terms are linearized by lagging appropriate factors. The modeling equations for the individual fluids are combined into a single system of equations using a continuum surface model. The numerical approximation is stabilized by using a SUPG approximation for the constitutive equation. Under a small data assumption on the true solution, existence of the approximate solution is proven. A priori error estimates for the approximation in terms of the mesh parameter $h$, the time discretization parameter $\Delta t$, and the SUPG coefficient $\nu$ are also derived. Numerical simulations of viscoelastic fluid flow involving two immiscible fluids are also presented.
\end{abstract}

Key words. viscoelasticity, finite element method, fully discrete, SUPG, multicomponent, interfacial tension

AMS Mathematics subject classifications. 65N30

\section{Introduction}

Presented in this paper is an error analysis for a fully discrete approximation to a time dependent, multicomponent, viscoelastic fluid flow problem. In [3] and [11], results are given for a fully discrete approximation to the flow of a single viscoelastic fluid. This paper extends these results to include flows which involve more than one fluid. Our motivation for considering multicomponent viscoelastic fluid flow arises from its application in material science, where new materials with novel properties are being developed by mixing several viscoelastic fluids [24].

For the governing equations of multicomponent viscoelastic fluid flow, we have that within each fluid component the viscoelastic equations must hold. In addition, along the interfaces separating components, a free-surface boundary condition must be satisfied. This boundary condition accounts for the discontinuity in the stress tensor across the interface between components and, in part, determines the shape of the interface. Using a continuum surface force model (CSF) [6], we replace

\footnotetext{
${ }^{*}$ Department of Mathematical Sciences, Clemson University, Clemson, South Carolina 29634-0975

${ }^{\dagger}$ Department of Mathematics and Computer Science, Stetson University, DeLand, Florida 32724

${ }^{\ddagger}$ This work was partially supported by the ERC Program of the National Science Foundation under Award Number ERC-9731680.
} 
the interfacial surface with an interfacial region in which we use a continuous interpolate to describe the fluid characteristics. The width of the interfacial region is determined a priori. The width is fixed and independent of the computational mesh. The CSF approach enables us to model and analyse the multicomponent fluid problem as a single fluid with varying material parameters.

In viscoelasticity, under a "slow flow" assumption, the non-linearity in the momentum equation is often neglected. For an Oldroyd B type fluid, the stress is defined by a differential constitutive equation. The difficulity in performing accurate numerical computations arises from the hyperbolic character of the constitutive equation. Care must be used in discretizing the constitutive equation to avoid the introduction of spurious oscillations into the approximation.

The first error analysis for the steady-state finite element approximation of viscoelastic fluid flow was presented by Baranger and Sandri [2]. In this paper a discontinuous finite element formulation was used for the discretization of the constitutive equation, with the approximation for the stress being discontinuous. Motivated by implementation considereation, Najib and Sandri in [17] modified the discretization in [2] to obtain a decoupled system of two equations, showed the algorithm was convergent, and gave error estimates. In [20], Sandri presented an analysis of a finite element approximation to this problem wherein the constitutive equation was discretized using a Streamline Upwind Petrov Galerkin (SUPG) method. For the constitutive equation discretized using the method of characteristics, Baranger and Machmoum in [1] analysed this approach and gave error estimates for the approximations.

In the analyses described above for steady-state viscoelastic flow there are three main steps: (i) the definition of a iteration operator, (ii) showing that the iteration operator is well defined, and (iii) applying Brouwer's fixed point theorem.

For the fully discrete approximation to the time dependent, multicomponent problem case presented herein, the analysis is completely different from the aformentioned method. Instead it follows closely the method of [11]. Time derivatives are replaced by backward difference quotients, and the nonlinear terms are linearized by lagging appropriate factors. A key part in the error analysis is an induction argument on properties of the approximation. The approach follows that of Liu [15] for compressible Navier-Stokes equations. For completeness we present the analysis with the non-linear term in the momentum equation included.

This paper is organized as follows. In Section 2, the general equations which govern the flow of multicomponent behavior are discussed, and the continuum surface force model is presented. In Section 2 we describe the equations for viscoelastic fluid flow and present the numerical approximation scheme. The main approximation result is then given in Theorem 4.1 in Section 4, followed by its proof. In Section 5 we present a numerical simulation of viscoelastic fluid flow involving two immiscible fluids. The experimental convergence rates for the error in the numerical simulation agree with the theoretical rates established in Section 4.

\section{The Modeling Equations of Multicomponent Fluid Flow}

In this section, we briefly present the modeling equations describing multicomponent, viscoelastic fluid flow. We use $\mathbf{u}, \rho$, and $\mathbf{T}$ to denote the velocity, density, and total stress (tensor) of the fluid.

Let $\Omega$ denote a bounded domain in $\Re^{d}(\dot{d}=2,3)$, with boundary $\partial \Omega$. For ease of exposition, we will 
present the formulation for two viscoelastic fluids in $\Omega$. Let $\Omega_{1}, \Omega_{2}$ denote the region in $\Omega$ occupied by fluids 1 and 2 , respectively, and $\mathcal{I}$, the interface between the two fluids. Note that $\Omega_{1}, \Omega_{2}$ and $\mathcal{I}$ are functions of time, and $\Omega=\Omega_{1} \cup \Omega_{2} \cup \mathcal{I}$.

Within each $\Omega_{i}$ :

For $V$ a fixed region in $\Omega_{i}$, with boundary $\partial V$, the conservation of momentum and mass equations imply

$$
\begin{aligned}
\frac{d}{d t} \int_{V} \rho \mathbf{u} d \mathbf{x} & =\int_{V} \mathbf{b} d \mathbf{x}+\int_{\partial V} \mathbf{T} \cdot \mathbf{n} d S-\int_{\partial V} \rho \mathbf{u}(\mathbf{u} \cdot \mathbf{n}) d S \\
\frac{d}{d t} \int_{V} \rho d \mathbf{x} & =-\int_{\partial V} \rho \mathbf{u} \cdot \mathbf{n} d S
\end{aligned}
$$

where $\mathbf{n}$ denotes the unit outward normal on $\partial V$, and $\mathbf{b}$ the body forces acting on $V$.

Along the Interface $\mathcal{I}$ :

The boundary condition which holds along, and determines the interface $\mathcal{I}$ is [4]

$$
[\mathbf{T} \cdot \mathbf{n}]=-\sigma \kappa \mathbf{n}-\nabla_{s} \sigma
$$

where $\kappa$ denotes the mean curvature of $\mathcal{I}, \sigma$ the coefficient of interfacial tension, $\nabla_{s} \sigma$ the surface gradient of $\sigma, \mathbf{n}$ the unit normal on $\mathcal{I}$ pointing into fluid 2 , and $[\mathbf{T} \cdot \mathbf{n}]$ the jump of the normal component of stress across $\mathcal{I}$ defined by

$$
\left.[\mathbf{T} \cdot \mathbf{n}]\right|_{\mathbf{x}}=\lim _{\epsilon \rightarrow 0^{+}}\left(\left.\mathbf{T}\right|_{\mathbf{x}+\epsilon \mathbf{n}}-\left.\mathbf{T}\right|_{\mathbf{x}-\epsilon \mathbf{n}}\right) .
$$

Using the continuum surface force model of Brackbill et. al. [6], the force along the interface is rewritten as a volume force using a delta distribution, i.e.

$$
\begin{aligned}
\int_{V \cap \mathcal{I}}[\mathbf{T} \cdot \mathbf{n}] d S & =\int_{V}[\mathbf{T} \cdot \mathbf{n}] \delta\left(\mathbf{x}-\mathbf{x}_{s}\right) \\
& =\int_{V}\left(-\sigma \kappa \mathbf{n}-\nabla_{s} \sigma\right) \delta\left(\mathbf{x}-\mathbf{x}_{s}\right) d \mathbf{x}
\end{aligned}
$$

where $\mathbf{x}_{s}$ denotes a nearest point to $\mathbf{x}$ on $\mathcal{I}$.

Using the divergence theorem to replace the surface integrals in (2.1), (2.2) with volume integrals, the fact that $V$ is an arbitrary volume, and the incompressibility of the fluid, we obtain the following pointwise equations for the conservation of momentum and mass:

$$
\begin{aligned}
\rho \frac{\partial \mathbf{u}}{\partial t}+\rho \mathbf{u} \cdot \nabla \mathbf{u} & =\mathbf{b}+\nabla \cdot \mathbf{T}-\left(\sigma \kappa \mathbf{n}+\nabla_{s} \sigma\right) \delta_{\mathcal{I}}, & & \text { in } \Omega, \\
\nabla \cdot \mathbf{u} & =0 & & \text { in } \Omega .
\end{aligned}
$$

Modeling Equation for the Stress Tensor T:

The stress tensor $\mathbf{T}$ is written in the form

$$
\mathbf{T}=-p I+\tau
$$


where $p$ denotes the internal fluid pressure, $I$ the identity tensor, and $\tau$ the extra stress tensor. For a Newtonian fluid $\tau$ is modeled as

$$
\tau=2 \eta D(\mathbf{u})
$$

where $D(\mathbf{u}):=\frac{1}{2}\left(\nabla \mathbf{u}+\nabla \mathbf{u}^{T}\right)$ is the deformation tensor and $\eta$ is the fluid viscosity. For viscoelastic fluids, because of the internal elasticity of the fluid, the modeling equation for the extra stress is in general considerably more complicated than (2.7), (see [5] for a description of various models).

In this paper we assume that the extra stress is governed by an Oldroyd B model. For this model $\tau$ is expressed as

$$
\tau=\tau_{n}+\tau_{v}
$$

where the Newtonian contribution to the extra stress, $\tau_{n}$ satisfies

$$
\tau_{n}=2(1-\alpha) D(\mathbf{u})
$$

and the viscoelastic contribution $\tau_{v}$ is given by

$$
\tau_{v}+\lambda \frac{\hat{\partial} \tau_{v}}{\partial t}-2 \alpha D(\mathbf{u})=0
$$

where

$$
\frac{\hat{\partial} \tau_{v}}{\partial t}:=\frac{\partial \tau_{v}}{\partial t}+\mathbf{u} \cdot \nabla \tau_{v}+g_{a}\left(\tau_{v}, \nabla \mathbf{u}\right), \quad a \in[-1,1]
$$

and

$$
g_{a}(\sigma, \nabla \mathbf{u}):=\frac{1-a}{2}\left(\tau_{v} \nabla \mathbf{u}+(\nabla \mathbf{u})^{T} \tau_{v}\right)-\frac{1+a}{2}\left((\nabla \mathbf{u}) \tau_{v}+\tau_{v}(\nabla \mathbf{u})^{T}\right)
$$

In (2.9), $\alpha \in(0,1)$ may be interpreted as the proportion of the viscosity which is considered to be viscoelastic in nature. The Weissenberg number, $\lambda$, is a dimensionless constant which is defined as the product of the relaxation time and a characteristic strain rate [5]. In (2.11) the choices $a=1,-1,0$ correspond to the upper, lower, and corrotational convected derivatives of $\tau_{v}$, respectively.

In what follows, for ease of notation, we use $\tau$ to denote $\tau_{v}$. Using (2.6), (2.8)-(2.11) and (2.4), (2.5) we obtain, on nondimensionalization of the problem, the modeling system of equations:

$$
\begin{aligned}
R e\left(\frac{\partial \mathbf{u}}{\partial t}+\mathbf{u} \cdot \nabla \mathbf{u}\right)+\nabla p-2(1-\alpha) \nabla \cdot D(\mathbf{u})-\nabla \cdot \tau & =\mathbf{f} & \text { in } \Omega \\
\tau+\lambda\left(\frac{\partial \tau}{\partial t}+\mathbf{u} \cdot \nabla \tau+g_{a}(\tau, \nabla \mathbf{u})\right)-2 \alpha D(\mathbf{u}) & =0 & \text { in } \Omega \\
\nabla \cdot \mathbf{u} & =0 & \text { in } \Omega
\end{aligned}
$$

where

$$
\begin{aligned}
\mathbf{f} & :=\mathbf{b}-\left(\sigma \kappa \mathbf{n}+\nabla_{s} \sigma\right) \tilde{\delta}_{\mathcal{I}} \\
R e & :=\frac{L V \bar{\rho}}{\bar{\eta}}
\end{aligned}
$$


In (2.16), $\tilde{\delta}_{\mathcal{I}}$ is a $C^{\infty}$ approximation to the dirac delta, $\delta$. The approximation is centered along the interface with a fixed width. More regarding the implementation of this approximation is given in the next subsection. In (2.17), $L, V, \bar{\rho}, \bar{\eta}$ denote a characteristic length scale, velocity scale, density, and viscosity.

To fully specify the problem, together with (2.13)-(2.15), we requre initial conditions for the velocity and stress, boundary conditions for the velocity, and the stress specified on the inflow boundary of $\Omega, \partial \Omega_{\text {in }}$,

$$
\begin{aligned}
\mathbf{u}(\mathbf{x}, 0) & =\mathbf{u}_{0}(\mathbf{x}) & & \text { in } \Omega, \\
\tau(\mathbf{x}, 0) & =\tau_{0}(\mathbf{x}) & & \text { in } \Omega . \\
\mathbf{u} & =\mathbf{u}_{\mathrm{bdy}} & & \text { on } \partial \Omega, \\
\tau & =\tau_{\text {bdy }} & & \text { on } \partial \Omega_{\text {in }} .
\end{aligned}
$$

Note: Equations (2.13)-(2.15), (2.18)-(2.21) only specify the pressure $p$ up to an arbitrary constant.

The existence and uniqueness of ( $\mathbf{u}, \tau, p)$ satisfying (2.13)-(2.15), (2.18)-(2.21) is still largely an open research question. The local existence (in time), and under a "small data" assumption on $\mathbf{f}, \mathbf{f}^{\prime}, \mathbf{u}_{0}, \tau_{0}$, global existence (in time) of solutions to (2.13)-(2.15), (2.18)-(2.21) have been established [13]. For a more complete discussion of existence and uniqueness issues, see [19].

In order to simplify the numerical analysis of the approximation scheme to (2.13)-(2.15), (2.18)(2.21), we will assume homogeneous boundary conditions for the velocity (i.e. $\mathbf{u}_{\text {bdy }}=0$ ). Consequently, as there is no inflow boundary, below we study the specific system of equations (2.13)-(2.15), (2.18)-(2.20) with $\mathbf{u}_{\text {bdy }}=0$.

\subsection{Interfacial Tension Forces}

There are a number of methods for tracking an interface. These methods can be broadly classified as front tracking [23], or front capturing methods [12, 21, 22]. We use a front capturing method commonly referred to as the level set method.

An equation describing the movement of the interface is the advection equation:

$$
\frac{\partial C}{\partial t}+\nabla \cdot(\mathbf{u} C)=0
$$

where $C$ is called the "color" function. The color function is an indicator function with respect to the fluids in the flow field. For a two fluid mixture the color function has a value of 1 in fluid 1 and a value of -1 in fluid 2 .

The level set method was first introduced and developed by Osher and Sethian in the late 1980's (see [18]). For the level set method a distance function, $\phi(\mathbf{x}, t)$, is used as the color function. Distance to the interface is taken to be positive within fluid 1 and negative within fluid 2 . Thus, the interface is given by $\phi(\mathbf{x}, t)=0$, i.e. the zero-level curve of $\phi(\mathbf{x} t)$.

To obtain a pointwise equation for the conservation of momentum we reformulate the interfacial tension forces, which are surface forces, as volume forces using the continuum surface force approach of Brackbill et. al. [6]. In this approach the interfacial surface is replaced by an interfacial region. 
The color function $C(x, t)$ is modified so as to change continuously from 1 (the color value in fluid 1) to -1 (the color value in fluid 2). The modified color function is denoted $\tilde{C}$. Using this modification, the two-component fluid system may be considered as a single fluid with continuous (nonconstant) fluid parameters. The interfacial force may be represented by a local volume force, $F_{s v}$, by multiplying by a $\delta$ distribution centered at the interface. In [6] Brackbill et. al. showed that

$$
\lim _{\epsilon \rightarrow 0} \int_{V} \sigma \kappa(x) \frac{\nabla \tilde{C}(x)}{[C]} d V=\int_{\mathcal{I}} \sigma \kappa \mathbf{n} d S
$$

where $\tilde{C}$ is a mollified version of $C,[C]=C_{2}-C_{1}, \epsilon$ is the half-width of the interfacial region. In addition, the normal is approximated by $\mathbf{n}(x)=\frac{\nabla \tilde{C}(x)}{|\tilde{C}(x)|}$.

Several analogs of (2.23) have been developed. We have extended a formulation derived by Chang in [8] to allow for a spatially varying coefficient of interfacial tension, $\sigma$. For the level set function denoted by $\phi$ we have:

Lemma 1 [16] The interfacial tension forces may be expressed as

$$
\int_{\mathcal{I}}\left(\sigma \kappa \mathbf{n}+\nabla_{s} \sigma\right) d S=\int_{V}\left(\sigma \kappa \nabla \phi+\nabla_{s} \sigma\right) \delta(\phi) d V
$$

where $\delta$ is the dirac delta.

Using (2.24) the conservation of momentum can be expressed as

$$
\frac{d}{d t} \int_{V} \rho \mathbf{u} d V=\int_{V} \rho \mathbf{b} d V+\int_{V} \nabla \cdot \mathbf{T} d V+\int_{V}\left(\sigma \kappa \nabla \phi+\nabla_{s} \sigma\right) \delta(\phi) d V .
$$

In the numerical implementation, $\delta$ is replaced by and approximation $\tilde{\delta}$ given by

$$
\tilde{\delta}_{\mathcal{I}}(x)= \begin{cases}\frac{1}{2}(1+\cos (\pi x / \epsilon)) / \epsilon & \text { if }|x|<\epsilon, \\ 0 & \text { otherwise }\end{cases}
$$

where $\epsilon$ is half the interfacial width. The curvature $\kappa(x)$ is approximated using

$$
\tilde{n}=\frac{\nabla \tilde{\phi}}{|\nabla \tilde{\phi}|}, \quad \kappa \approx-\nabla \cdot \tilde{n}
$$

where $\tilde{\phi}$ is a smoothed version of $\phi$.

\section{The Variational Formulation}

In this section, we develop the variational formulation of (2.13)-(2.15), (2.18)-(2.20). The following notation will be used. The $L^{2}(\Omega)$ norm and inner product will be denoted by $\|\cdot\|$ and $(\cdot, \cdot)$. Likewise, the $L^{p}(\Omega)$ norms and the Sobolev $W^{k, p}(\Omega)$ norms are denoted by $\|\cdot\|_{L^{p}}$ and $\|\cdot\|_{k, p}$, respectively. $H^{k}$ 
is used to represent the Sobelev space $W^{k, 2}$, and $\|\cdot\|_{k}$ denotes the norm in $H^{k}$. The following function spaces are used in the analysis:

$$
\begin{aligned}
& \text { Velocity Space : } X:=H_{0}^{1}(\Omega):=\left\{\mathbf{u} \in H^{1}(\Omega): \mathbf{u}=0 \text { on } \partial \Omega\right\} \text {, } \\
& \text { Stress Space }: S:=\left\{\tau=\left(\tau_{i j}\right): \tau_{i j}=\tau_{j i} ; \tau_{i j} \in L^{2}(\Omega) ; 1 \leq i, j \leq 3\right\} \\
& \cap\left\{\tau=\left(\tau_{i j}\right): \mathbf{u} \cdot \nabla \tau \in L^{2}(\Omega), \forall \mathbf{u} \in X\right\}, \\
& \text { Pressure Space : } Q:=L_{0}^{2}(\Omega)=\left\{q \in L^{2}(\Omega): \int_{\Omega} q d x=0\right\} \text {, } \\
& \text { Divergence - free Space }: Z:=\left\{v \in X: \int_{\Omega} q(\nabla \cdot v) d x=0, \forall q \in Q\right\} \text {. }
\end{aligned}
$$

The variational formulation of (2.13)-(2.15), (2.18)-(2.20) proceeds in the usual manner. Taking the inner product of (2.13), (2.14), and (2.15) with a velocity test function, a stress test function, and a pressure test function respectively, we obtain

$$
\begin{aligned}
\left(R e \frac{\partial \mathbf{u}}{\partial t}+R e \mathbf{u} \cdot \nabla \mathbf{u}, \mathbf{v}\right)-(p, \nabla \cdot \mathbf{v})+(2(1-\alpha) D(\mathbf{u})+\tau, D(\mathbf{v})) & =(\mathbf{f}, \mathbf{v}), & & \forall \mathbf{v} \in X, \\
\left(\tau+\left(\lambda \frac{\partial \tau}{\partial t}+\lambda \mathbf{u} \cdot \nabla \tau+\lambda g_{a}\left(\tau_{V}, \nabla \mathbf{u}\right)\right)-2 \alpha D(\mathbf{u}), \psi\right) & =0, & & \forall \psi \in S, \\
(\nabla \cdot \mathbf{u}, q) & =0, & & \forall q \in Q .
\end{aligned}
$$

Note that $R e$ and $\lambda$ are functions of time and space, determined by which fluid is occupying the point $\mathbf{x}$ at time $t$. We use

$$
\begin{aligned}
0<R e_{m} & :=\min _{\mathbf{x} \in \Omega} R e, \\
0<R e_{M} & :=\max _{\mathbf{x} \in \Omega} R e, \\
0<\lambda_{m} & :=\min _{\mathbf{x} \in \Omega} \lambda, \\
0<\lambda_{M} & :=\max _{\mathbf{x} \in \Omega} \lambda .
\end{aligned}
$$

The space $Z$ is the space of weakly divergence free functions. The condition

$$
(\nabla \cdot \mathbf{u}, q)=0, \quad \forall q \in Q, \mathbf{u} \in X
$$

is equivalent in a "distributional" sense to

$$
(\mathbf{u}, \nabla q)=0, \forall q \in Q, \mathbf{u} \in X,
$$

where in $(3.4),(\cdot, \cdot)$ denotes the duality pairing between $H^{-1}$ and $H_{0}^{1}$ functions. In addition, note that the velocity and pressure spaces, $X$ and $Q$, satisfy the inf-sup condition

$$
\inf _{q \in Q} \sup _{v \in X} \frac{(q, \nabla \cdot v)}{\|q\|\|v\|_{1}} \geq \beta>0 .
$$

Since the inf-sup condition (3.5) holds, an equivalent variational formulation to (3.1)-(3.3) is: find $\mathbf{u} \in Z, \tau \in S$ satisfying

$$
\begin{aligned}
\left(R e \frac{\partial \mathbf{u}}{\partial t}+\operatorname{Re} \mathbf{u} \cdot \nabla \mathbf{u}, \mathbf{v}\right)+(2(1-\alpha) D(\mathbf{u})+\tau, \mathbf{D}(\mathbf{v})) & =(\mathbf{f}, \mathbf{v}), \quad \forall \mathbf{v} \in Z, \\
\left(\tau+\lambda\left(\frac{\partial \tau}{\partial t}+\mathbf{u} \cdot \nabla \tau+g_{a}(\tau, \nabla \mathbf{u})\right)-2 \alpha D(\mathbf{u}), \psi\right) & =0, \quad \forall \psi \in S .
\end{aligned}
$$


We assume that the fluid flow satisfies the following properties:

$$
\|\mathbf{u}\|_{\infty},\|\tau\|_{\infty},\|\nabla \mathbf{u}\|_{\infty},\|\nabla \tau\|_{\infty} \leq M
$$

for all $t \in[0, T]$.

The following definitions are used in the analysis below:

$$
\begin{aligned}
b(\mathbf{u}, \tau, \psi) & :=(\mathbf{u} \cdot \nabla \tau, \psi) \\
c(\mathbf{w}, \mathbf{u}, \mathbf{v}) & :=(\mathbf{w} \cdot \nabla \mathbf{u}, \mathbf{v}) .
\end{aligned}
$$

\subsection{Finite Element Approximation}

In this section we formulate a fully discrete finite element method for solving the viscoelastic fluid flow equations, and prove the solvability of the approximation at each step (for sufficiently small $\Delta t$ and $h)$. We begin by describing the finite element approximation framework and listing the approximating properties and inverse estimates used in the analysis.

Let $T_{h}$ be a triangulation of $\Omega$ made of triangles (in $\mathbb{R}^{2}$ ) or tetrahedrals (in $\mathbb{R}^{3}$ ). Thus, the computational domain is defined by

$$
\Omega=\bigcup K ; \quad K \in T_{h} .
$$

We assume that there exist constants $c_{1}, c_{2}$ such that

$$
c_{1} h \leq h_{K} \leq c_{2} \rho_{K}
$$

where $h_{K}$ is the diameter of triangle (tetrahedral) $K, \rho_{K}$ is the diameter of the greatest ball (sphere) included in $K$, and $h=\max _{K \in T_{h}} h_{K}$. Let $P_{k}(K)$ denote the space of polynomials on $A$ of degree no greater than $k$. Then we define the finite element spaces as follows.

$$
\begin{aligned}
X_{h} & :=\left\{\mathbf{v} \in X \cap C(\bar{\Omega})^{2}:\left.\mathbf{v}\right|_{K} \in P_{k}(K), \forall K \in T_{h}\right\}, \\
S_{h} & :=\left\{\sigma \in S \cap C(\bar{\Omega})^{4}:\left.\sigma\right|_{K} \in P_{m}(K), \forall K \in T_{h}\right\}, \\
Q_{h} & :=\left\{q \in Q \cap C(\bar{\Omega}):\left.q\right|_{K} \in P_{q}(K), \forall K \in T_{h}\right\}, \\
Z_{h} & :=\left\{\mathbf{v} \in X_{h}:(q, \nabla \cdot \mathbf{v})=0, \forall q \in Q_{h}\right\} .
\end{aligned}
$$

We assume that the velocity and pressure spaces are chosen so as to satisfy the discrete inf-sup condition:

$$
\inf _{q \in Q_{h}} \sup _{v \in X_{h}} \frac{(q, \nabla \cdot v)}{\|q\|\|v\|_{1}} \geq \beta>0 .
$$

Let $\Delta t$ denote the step size for $t, t_{n}:=n \Delta t, n=0,1,2, \ldots, N$, and let

$$
d_{t} f^{n}:=\frac{f\left(t_{n}\right)-f\left(t_{n-1}\right)}{\Delta t} .
$$

We also define the following additional norms:

$$
\begin{aligned}
\|v \mid\|_{\infty, k} & :=\max _{1 \leq n \leq N}\left\|v^{n}\right\|_{k}, \\
\|v\|_{0, k} & :=\left(\sum_{n=1}^{N}\left\|v^{n}\right\|_{k}^{2} \Delta t\right)^{\frac{1}{2}} .
\end{aligned}
$$


When $v(\mathbf{x}, t)$ is defined on the entire time interval $(0, T)$, we use

$$
\begin{aligned}
\|v\|_{\infty, k} & :=\sup _{0<t<T}\|v(\cdot, t)\|_{k}, \\
\|v\|_{0, k} & :=\left(\int_{0}^{T}\|v(\cdot, t)\|_{k}^{2} d t\right)^{1 / 2} .
\end{aligned}
$$

In addition, we make use of the following approximation properties, $[9]$ :

$$
\begin{aligned}
& \inf _{\mathbf{v} \in X_{h}}\|\mathbf{u}-\mathbf{v}\| \leq C h^{k+1}\|\mathbf{u}\|_{k+1}, \quad \mathbf{u} \in H^{k+1}(\Omega)^{\dot{d}}, \\
& \inf _{\mathbf{v} \in X_{h}}\|\mathbf{u}-\mathbf{v}\|_{1} \leq C h^{k}\|\mathbf{u}\|_{k+1}, \quad \mathbf{u} \in H^{k+1}(\Omega)^{\dot{d}}, \\
& \inf _{\sigma \in S_{h}}\|\tau-\sigma\| \leq C h^{m+1}\|\tau\|_{m+1}, \quad \tau \in H^{m+1}(\Omega)^{\dot{d} \times \dot{d}}, \\
& \inf _{\sigma \in S_{h}}\|\tau-\sigma\|_{1} \leq C h^{m}\|\tau\|_{m+1}, \quad \tau \in H^{m+1}(\Omega)^{\dot{d} \times \dot{d}}, \\
& \inf _{r \in Q_{h}}\|p-r\| \leq C h^{q+1}\|p\|_{q+1}, \quad p \in H^{q+1}(\Omega) .
\end{aligned}
$$

The following inverse estimates, [9], are also used:

$$
\begin{aligned}
\left\|\mathbf{u}_{h}\right\|_{\infty} & \leq c h^{-\frac{\dot{d}}{2}}\left\|\mathbf{u}_{h}\right\| \quad \forall \mathbf{u}_{h} \in X_{h} \\
\left\|q_{h}\right\|_{\infty} & \leq c h^{-\frac{d}{2}}\left\|q_{h}\right\| \quad \forall q_{h} \in Q_{h} .
\end{aligned}
$$

To solve the time-dependent flow equations numerically, time derivatives are replaced by backward differences, and nonlinear terms are lagged. As we are assuming "slow flow", i.e. $R e \equiv O(1)$, we use a conforming finite element method to discretize the momentum equation. For the constitutive equation for stress, which is hyperbolic, we use a streamline upwind Petrov-Galerkin (SUPG) discretization to control the production of spurious oscillations in the approximation. The discrete approximating system of equations is then:

\section{Approximating System}

$\overline{\text { For } n=1,2, \ldots, N, \text { find } \mathbf{u}_{h}^{n}} \in Z_{h}, \tau_{\mathbf{h}}^{\mathbf{n}} \in S_{h}$ such that

$$
\begin{gathered}
\left(\operatorname{Re} d_{t} \mathbf{u}_{h}^{n}, \mathbf{v}\right)+c\left(\operatorname{Re} \mathbf{u}_{h}^{n-1}, \mathbf{u}_{h}^{n}, \mathbf{v}\right)+2(1-\alpha)\left(D\left(\mathbf{u}_{h}^{n}\right), D(\mathbf{v})\right)-(p, \nabla \cdot \mathbf{v}) \\
+\left(\tau_{h}^{n}, D(\mathbf{v})\right)=\left(\mathbf{f}^{n}, \mathbf{v}\right), \forall \mathbf{v} \in X_{h}, \\
\left(\tau_{h}^{n}, \tilde{\sigma}\right)+\left(\lambda d_{t} \tau_{h}^{n}, \sigma\right)+b\left(\lambda \mathbf{u}_{h}^{n-1}, \tau_{h}^{n}, \tilde{\sigma}\right)-2 \alpha\left(D\left(\mathbf{u}_{h}^{n}\right), \tilde{\sigma}\right) \\
=-\left(\lambda g_{a}\left(\tau_{h}^{n-1}, \nabla \mathbf{u}_{h}^{n-1}\right), \tilde{\sigma}\right), \forall \sigma \in S_{h}, \\
\left(\nabla \cdot \mathbf{u}_{h}^{n}, q\right)=0, \forall q \in Q_{h},
\end{gathered}
$$

where $\tilde{\sigma}:=\sigma+\nu \sigma_{u}^{n}, \sigma_{u}^{n}:=\mathbf{u}_{h}^{n-1} \cdot \nabla \sigma$, and $\nu$ is a small positive constant.

As the spaces $X_{h}, Q_{h}$ satisfy the inf-sup condition (3.11), solving (3.15) - (3.17) is equivalent to: For $n=1,2, \ldots, N$, find $\mathbf{u}_{h}^{n} \in Z_{h}, \tau_{\mathbf{h}}^{\mathbf{n}} \in S_{h}$ such that

$$
\left(\operatorname{Re} d_{t} \mathbf{u}_{h}^{n}, \mathbf{v}\right)+c\left(\operatorname{Re} \mathbf{u}_{h}^{n-1}, \mathbf{u}_{h}^{n}, \mathbf{v}\right)+2(1-\alpha)\left(D\left(\mathbf{u}_{h}^{n}\right), D(\mathbf{v})\right)
$$




$$
\begin{gathered}
+\left(\tau_{h}^{n}, D(\mathbf{v})\right)=\left(\mathbf{f}^{n}, \mathbf{v}\right), \forall \mathbf{v} \in Z_{h}, \\
\left(\tau_{h}^{n}, \tilde{\sigma}\right)+\left(\lambda d_{t} \tau_{h}^{n}, \sigma\right)+b\left(\lambda \mathbf{u}_{h}^{n-1}, \tau_{h}^{n}, \tilde{\sigma}\right)-2 \alpha\left(D\left(\mathbf{u}_{h}^{n}\right), \tilde{\sigma}\right) \\
=-\left(\lambda g_{a}\left(\tau_{h}^{n-1}, \nabla \mathbf{u}_{h}^{n-1}\right), \tilde{\sigma}\right), \forall \sigma \in S_{h}(3.1
\end{gathered}
$$

The parameter $\nu>0$ is used to suppress the production of spurious oscillations in the approximation. Note that for $\nu=0$ the discretization of the constitutive equation is the usual Galerkin method. The goal in choosing $\nu$ is to keep it as small as possible, but large enough to control the generation of catastrophic spurious oscillations in the approximate stress.

To ensure computability of the algorithm, we begin by showing that (3.18)-(3.19) is uniquely solvable for $\mathbf{u}_{h}$ and $\tau_{h}$ at each time step $\mathrm{n}$. We use the following induction hypothesis.

$$
\left\|\mathbf{u}_{h}^{n-1}\right\|_{\infty},\left\|\tau_{h}^{n-1}\right\|_{\infty} \leq K
$$

Lemma 2 Assume (IH1) is true. For sufficiently small step size $\Delta t$, there exists a unique solution $\left(\mathbf{u}_{h}^{n}, \tau_{h}^{n}\right) \in Z_{h} \times S_{h}$ satisfying (3.18)-(3.19).

Proof: For notational simplicity, in this proof we drop the subscript $h$ from the variables. Choosing $\mathbf{v}=\mathbf{u}_{h}^{n}, \sigma=\tau_{h}^{n}$, multiplying (3.15) by $2 \alpha$ and adding to (3.16) we obtain

$$
\begin{aligned}
a\left(\mathbf{u}^{n}, \tau^{n} ; \mathbf{u}^{n}, \tau^{n}\right)=2 \alpha\left(\mathbf{f}^{n}, \mathbf{u}^{n}\right)+ & 2 \alpha \frac{1}{\Delta t}\left(\operatorname{Re} \mathbf{u}^{n-1}, \mathbf{u}^{n}\right) \\
& -\left(\lambda g_{a}\left(\tau^{n-1}, \nabla \mathbf{u}^{n-1}\right), \tilde{\tau}^{n}\right)+\frac{1}{\Delta t}\left(\lambda \tau^{n-1}, \tau^{n}\right),
\end{aligned}
$$

where the bilinear form $a(\mathbf{u}, \tau ; \mathbf{v}, \sigma)$ is defined as:

$$
\begin{aligned}
a(\mathbf{u}, \tau ; \mathbf{v}, \sigma):= & \frac{2 \alpha}{\Delta t}(\operatorname{Re} \mathbf{u}, \mathbf{u})+2 \alpha c\left(\operatorname{Re} \mathbf{u}^{n-1}, \mathbf{u}, \mathbf{v}\right)+4 \alpha(1-\alpha)(D(\mathbf{u}), D(\mathbf{v}))+2 \alpha(\tau, D(\mathbf{v})) \\
& +(\tau, \tilde{\sigma})+\frac{1}{\Delta t}(\lambda \tau, \sigma)+b\left(\lambda \mathbf{u}^{n-1}, \tau, \sigma\right)+b\left(\lambda \mathbf{u}^{n-1}, \tau, \nu \mathbf{u}^{n-1} \cdot \nabla \sigma\right) \\
& -2 \alpha(D(\mathbf{u}), \sigma)-2 \alpha\left(D(\mathbf{u}), \nu \mathbf{u}^{n-1} \cdot \nabla \sigma\right) .
\end{aligned}
$$

Thus,

$$
\begin{aligned}
a(\mathbf{u}, \tau ; \mathbf{u}, \tau)= & \frac{2 \alpha}{\Delta t}(\operatorname{Re} \mathbf{u}, \mathbf{u})+2 \alpha c\left(\operatorname{Re} \mathbf{u}^{n-1}, \mathbf{u}, \mathbf{u}\right)+4 \alpha(1-\alpha)(D(\mathbf{u}), D(\mathbf{u}))+(\tau, \tau)+\nu\left(\tau, \tau_{u}\right) \\
& +\frac{1}{\Delta t}(\lambda \tau, \tau)+b\left(\lambda \mathbf{u}^{n-1}, \tau, \tau\right)+b\left(\lambda \mathbf{u}^{n-1}, \tau, \nu \tau_{u}\right)-2 \alpha \nu\left(D(\mathbf{u}), \tau_{u}\right) .
\end{aligned}
$$

We now estimate the terms in $a\left(\mathbf{u}^{n}, \tau^{n} ; \mathbf{u}^{n}, \tau^{n}\right)$. We have

$$
\begin{aligned}
\frac{2 \alpha}{\Delta t}\left(\operatorname{Re} \mathbf{u}^{n}, \mathbf{u}^{n}\right) & \geq \frac{2 \alpha R e_{m}}{\Delta t}\left\|\mathbf{u}^{n}\right\|^{2} \\
\left|2 \alpha c\left(\operatorname{Re} \mathbf{u}^{n-1}, \mathbf{u}^{n}, \mathbf{u}^{n}\right)\right| & =2 \alpha\left|\left(\operatorname{Re} \mathbf{u}^{n-1} \cdot \nabla \mathbf{u}^{n}, \mathbf{u}^{n}\right)\right| \\
& \leq 2 \alpha d^{\frac{1}{2}}\left\|\operatorname{Re} \mathbf{u}^{n-1}\right\|_{\infty}\left\|\nabla \mathbf{u}^{n}\right\|\left\|\mathbf{u}^{n}\right\| \\
& \leq 2 \alpha d^{\frac{1}{2}} C_{K} \operatorname{Re}_{M}\left\|\mathbf{u}^{n-1}\right\|_{\infty}\left\|D\left(\mathbf{u}^{n}\right)\right\| \quad \text { (using Korn's lemma) }
\end{aligned}
$$




$$
\begin{aligned}
& \leq \epsilon_{1}\left\|D\left(\mathbf{u}^{n}\right)\right\|^{2}+\frac{\alpha^{2} d K^{2} C_{K}^{2} R e_{M}^{2}}{\epsilon_{1}}\left\|\mathbf{u}^{n}\right\|^{2}, \\
4 \alpha(1-\alpha)\left(D\left(\mathbf{u}^{n}\right), D\left(\mathbf{u}^{n}\right)\right) & =4 \alpha(1-\alpha)\left\|D\left(\mathbf{u}^{n}\right)\right\|^{2}, \\
\left(\tau^{n}, \tau^{n}\right) & =\left\|\tau^{n}\right\|^{2}, \\
\left|\nu\left(\tau^{n}, \tau_{u}^{n}\right)\right| & \leq \nu\left\|\tau^{n}\right\|\left\|\tau_{u}^{n}\right\| \\
& \leq\left\|\tau^{n}\right\|^{2}+\frac{\nu^{2}}{4}\left\|\tau_{u}^{n}\right\|^{2} \\
\frac{1}{\Delta t}\left(\lambda \tau^{n}, \tau^{n}\right) & \geq \frac{\lambda_{m}}{\Delta t}\left\|\tau^{n}\right\|^{2}, \\
\left|b\left(\lambda \mathbf{u}^{n-1}, \tau^{n}, \tau^{n}\right)\right|=\left|\left(\lambda \mathbf{u}^{n-1} \cdot \nabla \tau^{n}, \tau^{n}\right)\right| & \leq \lambda_{M}\left\|\tau_{u}^{n}\right\|\left\|\tau^{n}\right\| \\
b\left(\lambda \mathbf{u}^{n-1}, \tau^{n}, \nu \tau_{u}^{n}\right) & =\left(\lambda \tau_{u}^{n}, \nu \tau_{u}^{n}\right) \\
& \geq \epsilon_{m} \nu\left\|\tau_{u}^{n}\right\|^{2}, \\
\left|2 \alpha \nu\left(D\left(\mathbf{u}^{n}\right), \tau_{u}^{n}\right)\right| & \leq 2 \alpha \nu\left\|D\left(\mathbf{u}^{n}\right)\right\|\left\|\tau_{u}^{n}\right\| \\
& \leq \epsilon_{3}\left\|D\left(\mathbf{u}^{n}\right)\right\|^{2}+\frac{\alpha^{2} \nu^{2}}{\epsilon_{3}}\left\|\tau_{u}^{n}\right\|^{2} .
\end{aligned}
$$

Applying these inequalities to the bilinear form $a(\cdot, \cdot ; \cdot, \cdot)$ yields

$$
\begin{aligned}
a\left(\mathbf{u}^{n}, \tau^{n} ; \mathbf{u}^{n}, \tau^{n}\right) \geq & \left(\frac{2 \alpha R e_{m}}{\Delta t}-\frac{\alpha^{2} d K^{2} C_{K}^{2} R e_{M}}{\epsilon_{1}}\right)\left\|\mathbf{u}^{n}\right\|^{2}+\left(4 \alpha(1-\alpha)-\epsilon_{1}-\epsilon_{3}\right)\|D(\mathbf{u})\|^{2} \\
& +\left(\frac{\lambda_{m}}{\Delta t}-\frac{\lambda_{M}^{2}}{4 \epsilon_{2}}\right)\left\|\tau^{n}\right\|^{2}+\left(\lambda_{m} \nu-\epsilon_{2}-\frac{\nu^{2}}{4}-\frac{\alpha^{2} \nu^{2}}{\epsilon_{3}}\right)\left\|\tau_{u}^{n}\right\|^{2}
\end{aligned}
$$

For

$$
\nu \leq \frac{2 \lambda_{m}(1-\alpha)}{1+3 \alpha}
$$

and choosing $\epsilon_{1}=\epsilon_{3}=\alpha(1-\alpha), \epsilon_{2}=\frac{\lambda_{m} \nu}{2}$, we have that for

$$
\Delta t<\min \left\{\frac{4 \epsilon_{2} \lambda_{m}}{\lambda_{M}^{2}}, \frac{2 \epsilon_{1} \alpha R e_{m}}{d K^{2} C_{K}^{2} \alpha^{2} R e_{M}}\right\},
$$

the bilinear form $a(\cdot, \cdot ; \cdot, \cdot)$ is positive. Hence, (3.20) has at most one solution. Since (3.20) is a finite dimensional linear system, the uniqueness of the solution implies the existence of the solution.

Lemma 3 (Discrete Gronwall's Lemma) [14] Let $\Delta t, H$, and $a_{n}, b_{n}, c_{n}, \gamma_{n}$, (for integers $n \geq$ 0 ), be nonnegative numbers such that

$$
a_{l}+\Delta t \sum_{n=0}^{l} b_{n} \leq \Delta t \sum_{n=0}^{l} \gamma_{n} a_{n}+\Delta t \sum_{n=0}^{l} c_{n}+H \quad \text { for } l \geq 0 .
$$


Suppose that $\Delta t \gamma_{n}<1$, for all $n$, and set $\sigma_{n}=\left(1-\Delta t \gamma_{n}\right)^{-1}$. Then,

$$
a_{l}+\Delta t \sum_{n=0}^{l} b_{n} \leq \exp \left(\Delta t \sum_{n=0}^{l} \sigma_{n} \gamma_{n}\right)\left\{\Delta t \sum_{n=0}^{l} c_{n}+H\right\} \quad \text { for } l \geq 0 .
$$

\section{A Priori Error Estimate}

In this section we analyze the error between the finite element approximation given by (3.15)-(3.17) and the true solution. A priori error estimates for the approximation are in Theorem 4.1.

Theorem 4.1 Assume that the system (3.1)-(3.3) has a solution $(\mathbf{u}, \tau, \mathbf{p}) \in C^{2}\left(0, T ; H^{k+1}\right) \times$ $C^{2}\left(0, T ; H^{m+1}\right) \times C\left(0, T ; H^{q+1}\right)$. In addition assume that $\Delta t, \nu \leq c h^{d / 2}$, and

$$
\|\mathbf{u}\|_{\infty},\|\mathbf{u}\|_{\infty},\|\tau\|_{\infty},\|\tau\|_{\infty} \leq M \text { for all } t \in[0, T] .
$$

Then, the finite element approximation given by (3.15)-(3.17) is convergent to the solution of (3.1)(3.3) on the interval $(0, T)$ as $\Delta t, h \rightarrow 0$. In addition, the approximation $\left(\mathbf{u}_{h}, \tau_{h}\right)$ satisfies the following error estimates:

$$
\begin{aligned}
\left\|\mathbf{u}_{h}-\mathbf{u}\right\|_{\infty, 0}+\left\|\tau_{h}-\tau \mid\right\|_{\infty, 0} & \leq \mathbf{F}(\Delta t, \nu, h) \\
\left\|\mathbf{u}_{h}-\mathbf{u}\right\|_{0,1}+\left\|\tau_{h}-\tau\right\|_{0,0} & \leq \mathbf{F}(\Delta t, \nu, h)
\end{aligned}
$$

where

$$
\begin{aligned}
\mathbf{F}(\Delta t, \nu, h)= & C\left(h^{k}\left\|\mathbf{u}\left|\left\|_{0, k+1}+h^{k+1}\right\| \mathbf{u}_{t}\right|\right\|_{0, k+1}\right) \\
& +C\left(h^{m}\|\mid \tau\|_{0, m+1}+h^{m+1}\left\|\tau_{t}\right\| \|_{0, m+1}\right) \\
& +C h^{q+1}\|p\|_{0, q+1}+C\left(h^{k+1}\|\mathbf{u}\|_{\infty, k+1}+h^{m+1}\|\tau\|_{\infty, m+1}\right) \\
& +C|\Delta t|\left(\left\|\mathbf{u}_{t}\right\|_{0,1}+\left\|\mathbf{u}_{t t}\right\|_{0,0}+\left\|\tau_{t}\right\|_{0,1}+\left\|\tau_{t t}\right\|_{0,0}\right) \\
& +C \nu\left(\left\|\tau_{t}\right\|_{0,1}+\left\|\tau_{t}\right\|_{\infty, 0}\right) .
\end{aligned}
$$

In order to establish the estimates (4.2)-(4.3), we begin by introducing the following notation. Let $\mathbf{u}^{n}=\mathbf{u}\left(t_{n}\right), \tau^{n}=\tau\left(t_{n}\right)$ represent the solution of (3.6)-(3.7) at time $t_{n}$, and $\mathbf{u}_{h}^{n}, \tau_{h}^{n}$ denote the solution of (3.15)-(3.16). Let $\left(\mathcal{U}^{n}, \mathcal{P}^{n}\right)$ denote the Stokes projection of $\left(\mathbf{u}^{n}, p^{n}\right)$ into $\left(Z_{h}, Q_{h}\right)$, and $\mathcal{T}^{n}$ a Clément interpolant of $\tau^{n},[10]$. We have the approximating properties:

$$
\begin{aligned}
\left\|\mathbf{u}^{n}-\mathcal{U}^{n}\right\| & \leq C h^{k+1}\left\|\mathbf{u}^{n}\right\|_{k+1}, \\
\left\|\tau^{n}-\mathcal{T}^{n}\right\| & \leq C h^{m+1}\left\|\tau^{n}\right\|_{m+1}, \\
\left\|p^{n}-\mathcal{P}^{n}\right\| & \leq C h^{q+1}\left\|p^{n}\right\|_{q+1}, \\
\left\|\nabla\left(\mathbf{u}^{n}-\mathcal{U}^{n}\right)\right\| & \leq C h^{k}\left\|\mathbf{u}^{n}\right\|_{k+1}, \\
\left\|\nabla\left(\tau^{n}-\mathcal{T}^{n}\right)\right\| & \leq C h^{m}\left\|\tau^{n}\right\|_{m+1} .
\end{aligned}
$$


From [7], we have the following results.

Lemma 4 : Let $\left\{T_{h}\right\}, 0<h \leq 1$, denote a quasi-uniform family of subdivisions of a polyhedral domain $\Omega \subset \mathbb{R}^{d}$. Let $(\hat{K}, P, N)$ be a reference finite element such that $P \subset W_{p}^{l}(\hat{K}) \cap W_{q}^{m}(\hat{K})$ where $1 \leq p \leq \infty, 1 \leq q \leq \infty$ and $0 \leq m \leq l$. For $K \in T_{h}$, let $\left(K, P_{K}, N_{K}\right)$ be the affine equivalent element, and $V_{h}=\left\{v: v\right.$ is measurable and $\left.\left.v\right|_{K} \in P_{K}, \forall K \in T_{h}\right\}$. Then there exists $C=C(l, p, q)$ such that

$$
\left(\sum_{K \in T_{h}}\|v\|_{W_{p}^{l}(K)}^{p}\right)^{1 / p} \leq C h^{m-l+\min \left(0, \frac{\dot{d}}{p}-\frac{\dot{d}}{q}\right)}\left(\sum_{K \in T_{h}}\|v\|_{W_{q}^{m}(K)}^{q}\right)^{1 / q}
$$

for all $v \in V_{h}$.

Note that it follows from (3.8) and inverse estimates, [7], that

$$
\left\|\mathcal{U}^{n}\right\|_{\infty},\left\|\nabla \mathcal{U}^{n}\right\|_{\infty} \leq \tilde{M} \approx M .
$$

Below, for simplicity, we take $\tilde{M}=M$.

Define $\mathbf{\Lambda}^{n}, \mathbf{E}^{n}, \boldsymbol{\Gamma}^{n}, \mathbf{F}^{n}, \epsilon_{u}, \epsilon_{\tau}$ as

$$
\begin{aligned}
\boldsymbol{\Lambda}^{n}=\mathbf{u}^{n}-\mathcal{U}^{n}, & \mathbf{E}^{n}=\mathcal{U}^{n}-\mathbf{u}_{h}^{n}, \\
\boldsymbol{\Gamma}^{n}=\tau^{n}-\mathcal{T}^{n}, & \mathbf{F}^{n}=\mathcal{T}^{n}-\tau_{h}^{n}, \\
\epsilon_{u}=\mathbf{u}-\mathbf{u}_{h}^{n}, & \epsilon_{\tau}=\tau-\tau_{h}^{n} .
\end{aligned}
$$

The proof of theorem 4.1 is established in three steps.

1. Prove a lemma, assuming two induction hypotheses.

2. Show that the induction hypotheses are true.

3. Prove the error estimates given in $(4.2),(4.3)$.

Step 1. We prove the following lemma.

Lemma 5 Under the induction hypothesis (IH1) and the additional assumption

$$
\sum_{n=1}^{l-1} \Delta t\left\|\nabla E^{n}\right\|_{\infty} \leq 1
$$

we have that

$$
\left\|\mathbf{E}^{l}\right\|^{2}+\left\|\mathbf{F}^{l}\right\|^{2} \leq G(\Delta t, h, \nu)
$$

where

$$
\begin{aligned}
G(\Delta t, h, \nu)= & C\left(h^{2 k}\|\mathbf{u} \mid\|_{0, k+1}^{2}+h^{2 k+2}\left\|\mathbf{u}_{t}\right\|_{0, k+1}^{2}\right)+C\left(h^{2 m}\|\tau\|_{0, m+1}^{2}+h^{2 m+2}\left\|\tau_{t}\right\|_{0, m+1}^{2}\right) \\
& +C h^{2 q+2}\|p\|_{0, q+1}^{2}+C|\Delta t|^{2}\left(\left\|\mathbf{u}_{t}\right\|_{0,1}^{2}+\left\|\mathbf{u}_{t t}\right\|_{0,0}^{2}+\left\|\tau_{t}\right\|_{0,1}^{2}+\left\|\tau_{t t}\right\|_{0,0}^{2}\right) \\
& +C \nu^{2}\left(\left\|\tau_{t}\right\|_{0,1}^{2}+\left\|\tau_{t}\right\|_{\infty, 0}^{2}\right) .
\end{aligned}
$$


Proof of lemma 5: From (3.6)-(3.7), we have that the true solution $(\mathbf{u}, \tau)$ satisfies

$$
\begin{aligned}
\left(R e d_{t} \mathbf{u}^{n}, \mathbf{v}\right)+ & c\left(\operatorname{Re} \mathbf{u}_{h}^{n-1}, \mathbf{u}^{n}, \mathbf{v}\right)+2(1-\alpha)\left(D\left(\mathbf{u}^{n}\right), D(\mathbf{v})\right)+\left(\tau^{n}, D(\mathbf{v})\right) \\
& =\left(\mathbf{f}^{n}, \mathbf{v}\right)+\left(p^{n}, \nabla \cdot \mathbf{v}\right)+R_{1}(\mathbf{v}), \forall \mathbf{v} \in Z_{h}, \\
\left(\lambda d_{t} \tau^{n}, \sigma\right)+ & b\left(\lambda \mathbf{u}_{h}^{n-1}, \tau^{n}, \tilde{\sigma}\right)-2 \alpha\left(D\left(\mathbf{u}^{n}\right), \tilde{\sigma}\right)+\left(\tau^{n}, \tilde{\sigma}\right) \\
= & -\left(\lambda g_{a}\left(\tau_{h}^{n-1}, \nabla \mathbf{u}_{h}^{n-1}\right), \tilde{\sigma}\right)+R_{2}(\sigma), \forall \sigma \in S_{h},
\end{aligned}
$$

where

$$
R_{1}(\mathbf{v}):=\left(\operatorname{Re} d_{t} \mathbf{u}^{n}, \mathbf{v}\right)-\left(\operatorname{Re} \mathbf{u}_{t}^{n}, \mathbf{v}\right)+c\left(\operatorname{Re} \mathbf{u}_{h}^{n-1}, \mathbf{u}^{n}, \mathbf{v}\right)-c\left(\operatorname{Re} \mathbf{u}^{n}, \mathbf{u}^{n}, \mathbf{v}\right)
$$

and

$$
\begin{aligned}
R_{2}(\sigma):= & \left(\lambda d_{t} \tau^{n}, \sigma\right)-\left(\lambda \tau_{t}^{n}, \sigma\right)-\left(\lambda \tau_{t}^{n}, \nu \sigma_{u}\right)+b\left(\lambda \mathbf{u}_{h}^{n-1}, \tau^{n}, \tilde{\sigma}\right) \\
& -b\left(\lambda \mathbf{u}^{n}, \tau^{n}, \tilde{\sigma}\right)+\left(\lambda g_{a}\left(\tau_{h}^{n-1}, \nabla \mathbf{u}_{h}^{n-1}\right), \tilde{\sigma}\right)-\left(\lambda g_{a}\left(\tau^{n}, \nabla \mathbf{u}^{n}\right), \tilde{\sigma}\right) .
\end{aligned}
$$

Subtracting (3.15)-(3.16) from (4.8)-(4.9) we obtain the following equations for $\epsilon_{u}$ and $\epsilon_{\tau}$ :

$$
\begin{aligned}
\left(R e d_{t} \epsilon_{u}, \mathbf{v}\right)+c\left(\operatorname{Re} \mathbf{u}_{h}^{n-1}, \epsilon_{u}, \mathbf{v}\right)+ & 2(1-\alpha)\left(D\left(\epsilon_{u}\right), D(\mathbf{v})\right)+\left(\epsilon_{\tau}, D(\mathbf{v})\right) \\
& =\left(p^{n}, \nabla \cdot \mathbf{v}\right)+R_{1}(\mathbf{v}), \forall \mathbf{v} \in Z_{h}, \\
\left(\lambda d_{t} \epsilon_{\tau}, \sigma\right)+b\left(\lambda \mathbf{u}_{h}^{n-1}, \epsilon_{\tau}, \tilde{\sigma}\right)-2 \alpha\left(D\left(\epsilon_{u}\right), \tilde{\sigma}\right)+ & \left(\epsilon_{\tau}, \tilde{\sigma}\right)=R_{2}(\sigma), \forall \sigma \in S_{h} .
\end{aligned}
$$

Substituting $\epsilon_{u}=\mathbf{E}^{n}+\boldsymbol{\Lambda}^{n}, \epsilon_{\tau}=\mathbf{F}^{n}+\Gamma^{n}, \mathbf{v}=\mathbf{E}^{n}, \sigma=\mathbf{F}^{n}$ into (4.10)-(4.11), we obtain $\left(\operatorname{Re} d_{t} \mathbf{E}^{n}, \mathbf{E}^{n}\right)+c\left(\operatorname{Re} \mathbf{u}_{h}^{n-1}, \mathbf{E}^{n}, \mathbf{E}^{n}\right)+2(1-\alpha)\left(D\left(\mathbf{E}^{n}\right), D\left(\mathbf{E}^{n}\right)\right)+\left(\mathbf{F}^{n}, D\left(\mathbf{E}^{n}\right)\right)=\mathcal{F}_{1}\left(\mathbf{E}^{n}\right)$,

$$
\left(\lambda d_{t} \mathbf{F}^{n}, \mathbf{F}^{n}\right)+b\left(\lambda \mathbf{u}_{h}^{n-1}, \mathbf{F}^{n}, \tilde{\mathbf{F}}^{n}\right)-2 \alpha\left(D\left(\mathbf{E}^{n}\right), \tilde{\mathbf{F}}^{n}\right)+\left(\mathbf{F}^{n}, \tilde{\mathbf{F}}^{n}\right)=\mathcal{F}_{2}\left(\mathbf{F}^{n}\right),
$$

where,

$$
\begin{aligned}
\mathcal{F}_{1}\left(\mathbf{E}^{n}\right)= & \left(p^{n}, \nabla \cdot \mathbf{E}^{n}\right)+R_{1}\left(\mathbf{E}^{n}\right)-\left(\operatorname{Re} d_{t} \boldsymbol{\Lambda}^{n}, \mathbf{E}^{n}\right)-c\left(\operatorname{Re} \mathbf{u}_{h}^{n-1}, \boldsymbol{\Lambda}^{n}, \mathbf{E}^{n}\right) \\
& -2(1-\alpha)\left(D\left(\boldsymbol{\Lambda}^{n}\right), D\left(\mathbf{E}^{n}\right)\right)-\left(\boldsymbol{\Gamma}^{n}, D\left(\mathbf{E}^{n}\right)\right), \\
\mathcal{F}_{2}\left(\mathbf{F}^{n}\right)= & R_{2}\left(\mathbf{F}^{n}\right)-\left(\lambda d_{t} \boldsymbol{\Gamma}^{n}, \mathbf{F}^{n}\right)-b\left(\lambda \mathbf{u}_{h}^{n-1}, \boldsymbol{\Gamma}^{n}, \tilde{\mathbf{F}}^{n}\right)+2 \alpha\left(D\left(\boldsymbol{\Lambda}^{n}\right), \tilde{\mathbf{F}}^{n}\right)-\left(\boldsymbol{\Gamma}^{n}, \tilde{\mathbf{F}}^{n}\right) .
\end{aligned}
$$

Note that

$$
\begin{aligned}
\left(\operatorname{Re} d_{t} \mathbf{E}^{n}, \mathbf{E}^{n}\right) & =\frac{1}{\Delta t}\left(\left(\operatorname{Re} \mathbf{E}^{n}, \mathbf{E}^{n}\right)-\left(R e \mathbf{E}^{n-1}, \mathbf{E}^{n}\right)\right) \\
& \geq \frac{1}{\Delta t}\left(\left\|R e^{1 / 2} \mathbf{E}^{n}\right\|^{2}-\left\|R e^{1 / 2} \mathbf{E}^{n}\right\|\left\|R e^{1 / 2} \mathbf{E}^{n-1}\right\|\right) \\
& \geq \frac{1}{2 \Delta t}\left(\left\|R e^{1 / 2} \mathbf{E}^{n}\right\|^{2}-\left\|R e^{1 / 2} \mathbf{E}^{n-1}\right\|^{2}\right) \\
& \geq \frac{R e_{n}^{*}}{2 \Delta t}\left(\left\|\mathbf{E}^{n}\right\|^{2}-\left\|\mathbf{E}^{n-1}\right\|^{2}\right),
\end{aligned}
$$

where $R e_{n}^{*}=R e_{m}$ or $R e_{M}$ depending on the sign of $\left(\left\|\mathbf{E}^{n}\right\|^{2}-\left\|\mathbf{E}^{n-1}\right\|^{2}\right)$. Similarly, $\left(\lambda d_{t} \mathbf{F}^{n}, \mathbf{F}^{n}\right) \geq$ $\frac{\lambda_{n}^{*}}{2 \Delta t}\left(\left\|\mathbf{F}^{n}\right\|^{2}-\left\|\mathbf{F}^{n-1}\right\|^{2}\right)$. Then, from (4.12), we have that

$$
\begin{array}{r}
\frac{1}{2 \Delta t}\left(\left\|\mathbf{E}^{n}\right\|^{2}-\left\|\mathbf{E}^{n-1}\right\|^{2}\right)+\frac{1}{R e_{n}^{*}} c\left(\operatorname{Re} \mathbf{u}_{h}^{n-1}, \mathbf{E}^{n}, \mathbf{E}^{n}\right)+\frac{1}{R e_{n}^{*}} 2(1-\alpha)\left(D\left(\mathbf{E}^{n}\right), D\left(\mathbf{E}^{n}\right)\right) \\
+\frac{1}{R e_{n}^{*}}\left(\mathbf{F}^{n}, D\left(\mathbf{E}^{n}\right)\right) \leq \frac{1}{R e_{n}^{*}} \mathcal{F}_{1}\left(\mathbf{E}^{n}\right) .
\end{array}
$$


Multiplying (4.14) by $R e_{m} \Delta t$ and summing from $n=1$ to $l$ gives

$$
\begin{array}{r}
\frac{R e_{m}}{2}\left(\left\|\mathbf{E}^{l}\right\|^{2}-\left\|\mathbf{E}^{0}\right\|^{2}\right)+\sum_{n=1}^{l}\left\{\frac{R e_{m} \Delta t}{R e_{n}^{*}} c\left(R e \mathbf{u}_{h}^{n-1}, \mathbf{E}^{n}, \mathbf{E}^{n}\right)+\frac{R e_{m} \Delta t}{R e_{n}^{*}} 2(1-\alpha)\left\|D\left(\mathbf{E}^{n}\right)\right\|^{2}\right. \\
\left.+\frac{R e_{m} \Delta t}{R e_{n}^{*}}\left(\mathbf{F}^{n}, D\left(\mathbf{E}^{n}\right)\right)\right\} \leq \sum_{n=1}^{l} \frac{R e_{m} \Delta t}{R e_{n}^{*}} \mathcal{F}_{1}\left(\mathbf{E}^{n}\right) .
\end{array}
$$

Similarly, from (4.13) we have that

$$
\begin{gathered}
\frac{\lambda_{m}}{2}\left(\left\|\mathbf{F}^{l}\right\|^{2}-\left\|\mathbf{F}^{0}\right\|^{2}\right)+\sum_{n=1}^{l} \frac{\lambda_{m} \Delta t}{\lambda_{n}^{*}}\left\{\left(\mathbf{F}^{n}, \tilde{\mathbf{F}}^{n}\right)+b\left(\lambda \mathbf{u}_{h}^{n-1}, \mathbf{F}^{n}, \tilde{\mathbf{F}}^{n}\right)-2 \alpha\left(D\left(\mathbf{E}^{n}\right), \tilde{\mathbf{F}}^{n}\right)\right\} \\
\leq \sum_{n=1}^{l} \frac{\lambda_{m} \Delta t}{\lambda_{n}^{*}} \mathcal{F}_{2}\left(\mathbf{F}^{n}\right) .
\end{gathered}
$$

Multiplying (4.15) by $2 \alpha$ and adding to (4.16) yields

$$
\begin{aligned}
\alpha R e_{m}\left(\left\|\mathbf{E}^{l}\right\|^{2}-\left\|\mathbf{E}^{0}\right\|^{2}\right) & +\frac{\lambda_{m}}{2}\left(\left\|\mathbf{F}^{l}\right\|^{2}-\left\|\mathbf{F}^{0}\right\|^{2}\right)+4 \alpha(1-\alpha) \sum_{n=1}^{l} \frac{R e_{m} \Delta t}{R e_{n}^{*}}\left\|D\left(\mathbf{E}^{n}\right)\right\|^{2} \\
+\nu \sum_{n=1}^{l} \frac{\lambda_{m}^{2} \Delta t}{\lambda_{n}^{*}}\left\|\mathbf{F}_{u}^{n}\right\|^{2} & +\sum_{n=1}^{l} \frac{\lambda_{m} \Delta t}{\lambda_{n}^{*}}\left\|\mathbf{F}^{n}\right\|^{2} \\
& \leq \Delta t \sum_{n=1}^{l} \frac{2 \alpha R e_{m}}{R e_{n}^{*}}\left(\mathcal{F}_{1}\left(\mathbf{E}^{n}\right)-c\left(\operatorname{Re} \mathbf{u}_{h}^{n-1}, \mathbf{E}^{n}, \mathbf{E}^{n}\right)-\left(\mathbf{F}^{n}, D\left(\mathbf{E}^{n}\right)\right)\right) \\
& +\Delta t \sum_{n=1}^{l} \frac{\lambda_{m}}{\lambda_{n}^{*}}\left(\mathcal{F}_{2}\left(\mathbf{F}^{n}\right)-b\left(\lambda \mathbf{u}_{h}^{n-1}, \mathbf{F}^{n}, \mathbf{F}^{n}\right)+2 \alpha\left(D\left(\mathbf{E}^{n}\right), \tilde{\mathbf{F}}^{n}\right)-\nu\left(\mathbf{F}^{n}, \mathbf{F}_{u}^{n}\right)\right)
\end{aligned}
$$

Noting that $R e_{m} \leq R e_{n}^{*} \leq R e_{M}, \lambda_{m} \leq \lambda_{n}^{*} \leq \lambda_{M}$, applying the triangle inequality to the right hand side of (4.17), we have that

$$
\begin{gathered}
\alpha R e_{m}\left(\left\|\mathbf{E}^{l}\right\|^{2}-\left\|\mathbf{E}^{0}\right\|^{2}\right)+\frac{\lambda_{m}}{2}\left(\left\|\mathbf{F}^{l}\right\|^{2}-\left\|\mathbf{F}^{0}\right\|^{2}\right)+4 \alpha(1-\alpha) \sum_{n=1}^{l} \frac{R e_{m} \Delta t}{R e_{M}}\left\|D\left(\mathbf{E}^{n}\right)\right\|^{2} \\
+\nu \sum_{n=1}^{l} \frac{\lambda_{m}^{2} \Delta t}{\lambda_{M}}\left\|\mathbf{F}_{u}^{n}\right\|^{2}+\sum_{n=1}^{l} \frac{\lambda_{m} \Delta t}{\lambda_{M}}\left\|\mathbf{F}^{n}\right\|^{2} \leq \Delta t \sum_{n=1}^{l}\left\{2 \alpha\left|c\left(\operatorname{Re} \mathbf{u}_{h}^{n-1}, \mathbf{E}^{n}, \mathbf{E}^{n}\right)\right|+\left|b\left(\lambda \mathbf{u}_{h}^{n-1}, \mathbf{F}^{n}, \mathbf{F}^{n}\right)\right|\right. \\
\left.+2 \alpha \nu\left|\left(D\left(\mathbf{E}^{n}\right), \mathbf{F}_{u}^{n}\right)\right|+2 \alpha\left(1-\frac{R e_{m}}{R e_{M}}\right)\left|\left(D\left(\mathbf{E}^{n}\right), \mathbf{F}^{n}\right)\right|+\nu\left|\left(\mathbf{F}^{n}, \mathbf{F}_{u}^{n}\right)\right|\right\} \\
+\Delta t \sum_{n=1}^{l}\left\{2 \alpha\left|\mathcal{F}_{1}\left(\mathbf{E}^{n}\right)\right|+\left|\mathcal{F}_{2}\left(\mathbf{F}^{n}\right)\right|\right\} .
\end{gathered}
$$

We now estimate each term on the right hand side of (4.18). For $c\left(\mathbf{u}_{h}^{n-1}, \mathbf{E}^{n}, \mathbf{E}^{n}\right)$ we have that

$$
\begin{aligned}
2 \alpha c\left(\operatorname{Re} \mathbf{u}_{h}^{n-1}, \mathbf{E}^{n}, \mathbf{E}^{n}\right) & \leq 2 \alpha R e_{M}\left|\left(\mathbf{u}_{h}^{n-1} \cdot \nabla \mathbf{E}^{n}, \mathbf{E}^{n}\right)\right| \\
& \leq 2 \alpha R e_{M}\left\|\mathbf{u}_{h}^{n-1} \cdot \nabla \mathbf{E}^{n}\right\|\left\|\mathbf{E}^{n}\right\|
\end{aligned}
$$




$$
\begin{aligned}
\leq & 2 \alpha R e_{M}\left\|\mathbf{u}_{h}^{n-1}\right\|_{\infty} d^{\frac{1}{2}}\left\|\nabla \mathbf{E}^{n}\right\|\left\|\mathbf{E}^{n}\right\| \\
\leq & 4 \alpha^{2} \operatorname{Re}_{M}^{2} \epsilon_{1}\left\|\nabla \mathbf{E}^{n}\right\|^{2}+\frac{d K^{2}}{4 \epsilon_{1}}\left\|\mathbf{E}^{n}\right\|^{2}, \text { using (IH1). } \\
\leq & 4 \alpha^{2} \operatorname{Re}_{M}^{2} C_{K}^{2} \epsilon_{1}\left\|D\left(\mathbf{E}^{n}\right)\right\|^{2}+\frac{\hat{d} K^{2}}{4 \epsilon_{1}}\left\|\mathbf{E}^{n}\right\|^{2}, \\
& \text { (using Korn's lemma) }
\end{aligned}
$$

Note that for $\mathbf{v}=0$ on $\partial \Omega$, applying Green's theorem we have

$$
\begin{aligned}
b(\mathbf{v}, \tau, \sigma) & =-b(\mathbf{v}, \sigma, \tau)-(\nabla \cdot \mathbf{v} \tau, \sigma), \\
\Rightarrow b(\mathbf{v}, \tau, \tau) & =-\frac{1}{2}(\nabla \cdot \mathbf{v} \tau, \tau) .
\end{aligned}
$$

Using (4.21),

$$
\begin{aligned}
\left|b\left(\lambda \mathbf{u}_{h}^{n-1}, \mathbf{F}^{n}, \mathbf{F}^{n}\right)\right| & \leq \frac{\lambda_{M}}{2}\left|\left(\nabla \cdot \mathbf{u}_{h}^{n-1} \mathbf{F}^{n}, \mathbf{F}^{n}\right)\right| \\
& =\frac{\lambda_{M}}{2}\left|\left(\nabla \cdot\left(\mathbf{u}_{h}^{n-1}-\mathcal{U}^{n-1}\right) \mathbf{F}^{n}, \mathbf{F}^{n}\right)+\left(\nabla \cdot \mathcal{U}^{n-1} \mathbf{F}^{n}, \mathbf{F}^{n}\right)\right| \\
& \leq \frac{\lambda_{M}}{2}\left\|\nabla \cdot \mathbf{E}^{n-1}\right\|_{\infty}\left\|\mathbf{F}^{n}\right\|^{2}+\frac{\lambda_{M}}{2}\left\|\nabla \cdot \mathcal{U}^{n-1}\right\|_{\infty}\left\|\mathbf{F}^{n}\right\|^{2} \\
& \leq \frac{\lambda_{M}}{2}\left(\dot{d}\left\|\nabla \cdot \mathbf{E}^{n-1}\right\|_{\infty}\left\|\mathbf{F}^{n}\right\|^{2}+M\left\|\mathbf{F}^{n}\right\|^{2}\right), \text { using (4.6). }
\end{aligned}
$$

Next, with $\tilde{R}=\left(1-R e_{m} / R e_{M}\right)$,

$$
\begin{aligned}
2 \alpha \tilde{R}\left|\left(D\left(\mathbf{E}^{n}\right), \mathbf{F}^{n}\right)\right| & \leq 2 \alpha \tilde{R}\left\|D\left(\mathbf{E}^{n}\right)\right\|\left\|\mathbf{F}^{n}\right\| \\
& \leq 4 \alpha^{2} \tilde{R}^{2} \epsilon_{2}\left\|D\left(\mathbf{E}^{n}\right)\right\|^{2}+\frac{1}{4 \epsilon_{2}}\left\|\mathbf{F}^{n}\right\|^{2} .
\end{aligned}
$$

Then

$$
\begin{aligned}
2 \alpha\left|\left(D\left(\mathbf{E}^{n}\right), \nu \mathbf{F}_{u}^{n}\right)\right| & \leq 2 \alpha\left\|D\left(\mathbf{E}^{n}\right)\right\|\left\|\nu \mathbf{F}_{u}^{n}\right\| \\
& \leq 4 \alpha^{2} \epsilon_{3}\left\|D\left(\mathbf{E}^{n}\right)\right\|^{2}+\frac{\nu^{2}}{4 \epsilon_{3}}\left\|\mathbf{F}_{u}^{n}\right\|^{2} .
\end{aligned}
$$

Also,

$$
\begin{aligned}
\left|\left(\mathbf{F}^{n}, \nu \mathbf{F}_{u}^{n}\right)\right| & =\nu\left|\left(\mathbf{F}^{n}, \mathbf{F}_{u}^{n}\right)\right| \\
& \leq \nu\left\|\mathbf{F}^{n}\right\|\left\|\mathbf{F}_{u}^{n}\right\| \\
& \leq\left\|\mathbf{F}^{n}\right\|^{2}+\frac{\nu^{2}}{4}\left\|\mathbf{F}_{u}^{n}\right\|^{2}
\end{aligned}
$$

Thus, for the first summation on the right hand side of (4.18), we have

$$
\begin{aligned}
& \Delta t \sum_{n=1}^{l}\left\{2 \alpha\left|c\left(\operatorname{Re} \mathbf{u}_{h}^{n-1}, \mathbf{E}^{n}, \mathbf{E}^{n}\right)\right|+\left|b\left(\lambda \mathbf{u}_{h}^{n-1}, \mathbf{F}^{n}, \mathbf{F}^{n}\right)\right|+2 \alpha \nu\left|\left(D\left(\mathbf{E}^{n}\right), \mathbf{F}_{u}^{n}\right)\right|\right. \\
& \left.\quad-2 \alpha \tilde{R}\left|\left(D\left(\mathbf{E}^{n}\right), \mathbf{F}^{n}\right)\right|+\nu\left|\left(\mathbf{F}^{n}, \mathbf{F}_{u}^{n}\right)\right|\right\} \leq \Delta t \sum_{n=1}^{l}\left(4 \alpha^{2}\left(C_{K}^{2} R e_{M}^{2} \epsilon_{1}+\tilde{R}^{2} \epsilon_{2}+\epsilon_{3}\right)\right)\left\|D\left(\mathbf{E}^{n}\right)\right\|^{2}
\end{aligned}
$$




$$
\begin{aligned}
& +\Delta t \sum_{n=1}^{l}\left(\frac{\dot{d} K^{2}}{4 \epsilon_{1}}\right)\left\|\mathbf{E}^{n}\right\|^{2}+\Delta t \sum_{n=1}^{l}\left(\frac{\lambda_{M}}{2}\left(\dot{d}\left\|\nabla \mathbf{E}^{n-1}\right\|_{\infty}+M\right)+\frac{1}{4 \epsilon_{2}}+1\right)\left\|\mathbf{F}^{n}\right\|^{2} \\
& +\Delta t \sum_{n=1}^{l}\left(\frac{\nu^{2}}{4 \epsilon_{3}}+\frac{\nu^{2}}{4}\right)\left\|\mathbf{F}_{u}^{n}\right\|
\end{aligned}
$$

Next we consider $\mathcal{F}_{1}\left(\mathbf{E}^{n}\right)$.

$$
\begin{aligned}
& \left|\left(p^{n}, \nabla \cdot \mathbf{E}^{n}\right)\right|=\left|\left(p^{n}-\mathcal{P}^{n}, \nabla \cdot \mathbf{E}^{n}\right)\right| \\
& \leq\left\|p^{n}-\mathcal{P}^{n}\right\| d^{\frac{1}{2}}\left\|\nabla \mathbf{E}^{n}\right\| \\
& \leq C_{K}^{2} \epsilon_{5}\left\|D\left(\mathbf{E}^{n}\right)\right\|^{2}+\frac{\dot{d}}{4 \epsilon_{5}}\left\|p^{n}-\mathcal{P}^{n}\right\| \text {,(Korn's lemma). } \\
& \left|\left(R e d_{t} \mathbf{\Lambda}^{n}, \mathbf{E}^{n}\right)\right| \leq R e_{M}\left\|\mathbf{E}^{n}\right\|\left\|d_{t} \mathbf{\Lambda}^{n}\right\| \\
& \leq R e_{M}^{2}\left\|\mathbf{E}^{n}\right\|^{2}+\frac{1}{4}\left\|d_{t} \boldsymbol{\Lambda}^{n}\right\|^{2} . \\
& \left|c\left(R e \mathbf{u}_{h}^{n-1}, \boldsymbol{\Lambda}^{n}, \mathbf{E}^{n}\right)\right| \leq R e_{M}\left\|\mathbf{E}^{n}\right\|\left\|\mathbf{u}_{h}^{n-1} \cdot \nabla \boldsymbol{\Lambda}^{n}\right\| \\
& \leq R e_{M}\left\|\mathbf{E}^{n}\right\|\left\|\mathbf{u}_{h}^{n-1}\right\|_{\infty} \dot{d}^{\frac{1}{2}}\left\|\nabla \boldsymbol{\Lambda}^{n}\right\| \\
& \leq \quad \operatorname{Re}_{M}^{2}\left\|\mathbf{E}^{n}\right\|^{2}+\frac{K^{2} \dot{d}}{4}\left\|\nabla \boldsymbol{\Lambda}^{n}\right\|^{2}, \quad \text { using }(I H 1) . \\
& 2(1-\alpha)\left|\left(D\left(\boldsymbol{\Lambda}^{n}\right), D\left(\mathbf{E}^{n}\right)\right)\right| \leq(1-\alpha) \epsilon_{6}\left\|D\left(\mathbf{E}^{n}\right)\right\|^{2}+\frac{1-\alpha}{\epsilon_{6}}\left\|D\left(\boldsymbol{\Lambda}^{n}\right)\right\|^{2} . \\
& \left|\left(\boldsymbol{\Gamma}^{n}, D\left(\mathbf{E}^{n}\right)\right)\right| \leq\left\|D\left(\mathbf{E}^{n}\right)\right\|\left\|\boldsymbol{\Gamma}^{n}\right\| \\
& \leq \epsilon_{7}\left\|D\left(\mathbf{E}^{n}\right)\right\|^{2}+\frac{1}{4 \epsilon_{7}}\left\|\boldsymbol{\Gamma}^{n}\right\|^{2} .
\end{aligned}
$$

For the $R_{1}\left(\mathbf{E}^{n}\right)$ terms we have:

$$
\begin{aligned}
\left|\left(\operatorname{Re} d_{t} \mathbf{u}^{n}, \mathbf{E}^{n}\right)-\left(\operatorname{Re} \mathbf{u}_{t}^{n}, \mathbf{E}^{n}\right)\right| \leq & \operatorname{Re}_{M}^{2}\left\|\mathbf{E}^{n}\right\|^{2}+\frac{1}{4}\left\|d_{t} \mathbf{u}^{n}-\mathbf{u}_{t}^{n}\right\|^{2} . \\
\left|c\left(\operatorname{Re} \mathbf{u}_{h}^{n-1}, \mathbf{u}^{n}, \mathbf{E}^{n}\right)-c\left(\operatorname{Re} \mathbf{u}^{n}, \mathbf{u}^{n}, \mathbf{E}^{n}\right)\right|= & \mid c\left(\operatorname{Re}\left(\mathbf{u}_{h}^{n-1}-\mathcal{U}^{n-1}\right), \mathbf{u}^{n}, \mathbf{E}^{n}\right)+c\left(\operatorname{Re}\left(\mathcal{U}^{n-1}-\mathbf{u}^{n-1}\right), \mathbf{u}^{n}, \mathbf{E}^{n}\right) \\
& +c\left(\operatorname{Re}\left(\mathbf{u}^{n-1}-\mathbf{u}^{n}\right), \mathbf{u}^{n}, \mathbf{E}^{n}\right) \mid \\
\leq & \operatorname{Re} e_{M}\left\|\mathbf{E}^{n-1} \cdot \nabla \mathbf{u}^{n}\right\|\left\|\mathbf{E}^{n}\right\|+\operatorname{Re} M\left\|\mathbf{\Lambda}^{n-1} \cdot \nabla \mathbf{u}^{n}\right\|\left\|\mathbf{E}^{n}\right\| \\
& +\operatorname{Re}_{M}\left\|\left(\mathbf{u}^{n}-\mathbf{u}^{n-1}\right) \cdot \nabla \mathbf{u}^{n}\right\|\left\|\mathbf{E}^{n}\right\| \\
\leq & \operatorname{Re}_{M} \dot{d} M\left\|\mathbf{E}^{n-1}\right\|\left\|\mathbf{E}^{n}\right\|+\operatorname{Re}_{M} \dot{d} M\left\|\mathbf{\Lambda}^{n-1}\right\|\left\|\mathbf{E}^{n}\right\| \\
& +\operatorname{Re}_{M} \dot{d} M\left\|\left(\mathbf{u}^{n}-\mathbf{u}^{n-1}\right)\right\|\left\|\mathbf{E}^{n}\right\|, \operatorname{using}(3.8) \\
\leq & \frac{\operatorname{Re}_{M}^{2} \dot{d}^{2} M^{2}}{2}\left\|\mathbf{E}^{n-1}\right\|^{2}+\frac{3}{2}\left\|\mathbf{E}^{n}\right\|^{2}+\frac{\operatorname{Re}_{M^{2}}^{2} \dot{d}^{2} M^{2}}{2}\left\|\mathbf{\Lambda}^{n-1}\right\|^{2} \\
& +\frac{\operatorname{Re}_{M}^{2} \dot{d}^{2} M^{2}}{2} \Delta t \int_{t^{n-1}}^{t^{n}}\left\|\mathbf{u}_{t}\right\|^{2} d t .
\end{aligned}
$$

Combining (4.23)-(4.28) we have the following estimate for $\mathcal{F}_{1}\left(\mathbf{E}^{n}\right)$ :

$$
\left|2 \alpha \mathcal{F}_{1}\left(\mathbf{E}^{n}\right)\right| \leq 2 \alpha\left(C_{K}^{2} \epsilon_{5}+(1-\alpha) \epsilon_{6}+\epsilon_{7}\right)\left\|D\left(\mathbf{E}^{n}\right)\right\|^{2}+2 \alpha\left(3 R e_{M}^{2}+\frac{3}{2}\right)\left\|\mathbf{E}^{n}\right\|^{2}
$$




$$
\begin{aligned}
& +\alpha \operatorname{Re}_{M}^{2} \hat{d}^{2} M^{2}\left\|\mathbf{E}^{n-1}\right\|^{2}+2 \alpha \frac{\dot{d}}{4 \epsilon_{5}}\left\|\left(p^{n}-\mathcal{P}^{n}\right)\right\|^{2}+\alpha R e_{M}^{2} \hat{d}^{2} M^{2}\left\|\boldsymbol{\Lambda}^{n-1}\right\|^{2} \\
& +2 \alpha\left(\frac{K^{2} \dot{d}}{4}+\frac{1-\alpha}{\epsilon_{6}}\right)\left\|\nabla \boldsymbol{\Lambda}^{n}\right\|^{2}+\alpha \frac{1}{2}\left\|d_{t} \boldsymbol{\Lambda}^{n}\right\|^{2}+\alpha \frac{1}{2 \epsilon_{7}}\left\|\boldsymbol{\Gamma}^{n}\right\|^{2} \\
& +\alpha \frac{1}{2}\left\|d_{t} \mathbf{u}^{n}-\mathbf{u}_{t}^{n}\right\|^{2}+\alpha \operatorname{Re}_{M}^{2} \hat{d}^{2} M^{2} \Delta t \int_{t^{n-1}}^{t^{n}}\left\|\mathbf{u}_{t}\right\|^{2} d t
\end{aligned}
$$

Next we consider the terms in $\mathcal{F}_{2}\left(\mathbf{F}^{n}\right)$.

$$
\begin{aligned}
\left|\left(\lambda d_{t} \boldsymbol{\Gamma}^{n}, \mathbf{F}^{n}\right)\right| & \leq \lambda_{M}^{2}\left\|\mathbf{F}^{n}\right\|^{2}+\frac{1}{4}\left\|d_{t} \boldsymbol{\Gamma}^{n}\right\|^{2} . \\
\left|b\left(\lambda \mathbf{u}_{h}^{n-1}, \boldsymbol{\Gamma}^{n}, \tilde{\mathbf{F}}^{n}\right)\right| & =\left|b\left(\lambda \mathbf{u}_{h}^{n-1}, \boldsymbol{\Gamma}^{n}, \mathbf{F}^{n}\right)+b\left(\lambda \mathbf{u}_{h}^{n-1}, \boldsymbol{\Gamma}^{n}, \nu \mathbf{F}_{u}^{n}\right)\right| \\
& \leq \lambda_{M}\left\|\mathbf{u}_{h}^{n-1} \cdot \nabla \boldsymbol{\Gamma}^{n}\right\|\left\|\mathbf{F}^{n}\right\|+\lambda_{M}\left\|\mathbf{u}_{h}^{n-1} \cdot \nabla \boldsymbol{\Gamma}^{n}\right\|\left\|\nu \mathbf{F}_{u}^{n}\right\| \\
& \leq \lambda_{M} d^{\frac{1}{2}}\left\|\mathbf{u}_{h}^{n-1}\right\|_{\infty}\left\|\nabla \boldsymbol{\Gamma}^{n}\right\|\left\|\mathbf{F}^{n}\right\|+\lambda_{M} d^{\frac{1}{2}}\left\|\mathbf{u}_{h}^{n-1}\right\|_{\infty}\left\|\nabla \boldsymbol{\Gamma}^{n}\right\|\left\|\nu \mathbf{F}_{u}^{n}\right\| \\
& \leq \lambda_{M}^{2}\left\|\mathbf{F}^{n}\right\|^{2}+\nu^{2}\left\|\mathbf{F}_{u}^{n}\right\|^{2}+\frac{d K^{2}}{2}\left\|\nabla \boldsymbol{\Gamma}^{n}\right\|^{2} . \\
2 \alpha\left|\left(D\left(\boldsymbol{\Lambda}^{n}\right), \tilde{\mathbf{F}}^{n}\right)\right| & =2 \alpha\left|\left(D\left(\boldsymbol{\Lambda}^{n}\right), \mathbf{F}^{n}\right)+\left(D\left(\boldsymbol{\Lambda}^{n}\right), \nu \mathbf{F}_{u}^{n}\right)\right| . \\
& \leq\left\|\mathbf{F}^{n}\right\|^{2}+\nu^{2}\left\|\mathbf{F}_{u}^{n}\right\|^{2}+2 \alpha^{2}\left\|\nabla \boldsymbol{\Lambda}^{n}\right\|^{2} . \\
\left|\left(\boldsymbol{\Gamma}^{n}, \tilde{\mathbf{F}}^{n}\right)\right| & =\left|\left(\boldsymbol{\Gamma}^{n}, \mathbf{F}^{n}\right)+\nu\left(\boldsymbol{\Gamma}^{n}, \nu \mathbf{F}_{u}^{n}\right)\right| \\
& \leq\left\|\mathbf{F}^{n}\right\|^{2}+\nu^{2}\left\|\mathbf{F}_{u}^{n}\right\|^{2}+\frac{1}{2}\left\|\boldsymbol{\Gamma}^{n}\right\|^{2} .
\end{aligned}
$$

For the terms making up $R_{2}\left(\mathbf{F}^{n}\right)$ we have:

$$
\begin{aligned}
& \left|\left(\lambda d_{t} \tau^{n}, \mathbf{F}^{n}\right)-\left(\lambda \tau_{t}^{n}, \mathbf{F}^{n}\right)\right| \leq\left\|\lambda \mathbf{F}^{n}\right\|\left\|d_{t} \tau^{n}-\tau_{t}^{n}\right\| \\
& \leq \lambda_{M}^{2}\left\|\mathbf{F}^{n}\right\|^{2}+\frac{1}{4}\left\|d_{t} \tau^{n}-\tau_{t}^{n}\right\|^{2} . \\
& \left|\left(\lambda \tau_{t}^{n}, \nu \mathbf{F}_{u}^{n}\right)\right|=\left|\left(\lambda \tau_{t}^{n}, \nu \mathbf{u}_{h}^{n-1} \cdot \nabla \mathbf{F}^{n}\right)\right| \\
& =\left|B\left(\lambda \nu \mathbf{u}_{h}^{n-1}, \mathbf{F}^{n}, \tau_{t}^{n}\right)\right| \\
& \leq\left|B\left(\lambda \nu \mathbf{u}_{h}^{n-1}, \tau_{t}^{n}, \mathbf{F}^{n}\right)\right|+\left|\left(\nabla \cdot \mathbf{u}_{h}^{n-1} \lambda \nu \mathbf{F}^{n}, \tau_{t}^{n}\right)\right| \quad(\operatorname{using}(4.20)) \\
& \leq \lambda_{M} \nu\left\|\mathbf{u}_{h}^{n-1} \cdot \nabla \tau_{t}^{n}\right\|\left\|\mathbf{F}^{n}\right\|+\left|\left(\nabla \cdot\left(\mathbf{u}_{h}^{n-1}-\mathcal{U}^{n-1}\right) \lambda \nu \mathbf{F}^{n}, \tau_{t}^{n}\right)\right| \\
& +\left|\left(\nabla \cdot \mathcal{U}^{n-1} \lambda \nu \mathbf{F}^{n}, \tau_{t}^{n}\right)\right| \\
& \leq \lambda_{M} \nu\left\|\mathbf{u}_{h}^{n-1}\right\|_{\infty} \dot{d}^{\frac{1}{2}}\left\|\nabla \tau_{t}^{n}\right\|\left\|\mathbf{F}^{n}\right\|+\lambda_{M} \nu\left\|\nabla \cdot\left(\mathbf{u}_{h}^{n-1}-\mathcal{U}^{n-1}\right)\right\|_{\infty}\left\|\mathbf{F}^{n}\right\|\left\|\tau_{t}^{n}\right\| \\
& +\left\|\nabla \cdot \mathcal{U}^{n-1}\right\|_{\infty} \lambda_{M} \nu\left\|\mathbf{F}^{n}\right\|\left\|\tau_{t}^{n}\right\| \\
& \leq \lambda_{M}^{2}\left(2+\dot{d}\left\|\nabla \mathbf{E}^{n-1}\right\|_{\infty}\right)\left\|\mathbf{F}^{n}\right\|^{2}+\frac{\nu^{2}}{4}\left(\dot{d}^{2} M^{2}+\dot{d}\left\|\nabla \mathbf{E}^{n-1}\right\|_{\infty}\right)\left\|\tau_{t}^{n}\right\|^{2} \\
& +\frac{\nu^{2}}{4} K^{2} \dot{d}\left\|\nabla \tau_{t}\right\|^{2}, \quad(\text { using (4.6) and (IH1) ) . } \\
& \left|b\left(\lambda \mathbf{u}_{h}^{n-1}, \tau^{n}, \tilde{\mathbf{F}}^{n}\right)-b\left(\lambda \mathbf{u}^{n}, \tau^{n}, \tilde{\mathbf{F}}^{n}\right)\right|=\left|\left(\lambda\left(\mathbf{u}_{h}^{n-1}-\mathbf{u}^{n}\right) \cdot \nabla \tau^{n}, \tilde{\mathbf{F}}^{n}\right)\right|
\end{aligned}
$$




$$
\begin{aligned}
\leq & \left\|\left(\mathbf{u}_{h}^{n-1}-\mathbf{u}^{n}\right) \cdot \nabla \tau^{n}\right\|\left\|\lambda \tilde{\mathbf{F}}^{n}\right\| \\
\leq & \frac{\lambda_{M}^{2}}{2}\left\|\tilde{\mathbf{F}}^{n}\right\|^{2}+\frac{1}{2} \tilde{d}^{3}\left\|\nabla \tau^{n}\right\|_{\infty}^{2}\left\|\mathbf{u}_{h}^{n-1}-\mathbf{u}^{n}\right\|^{2} \\
\leq & \lambda_{M}^{2}\left\|\mathbf{F}^{n}\right\|^{2}+\lambda_{M}^{2} \nu^{2}\left\|\mathbf{F}_{u}^{n}\right\|^{2}+\frac{1}{2} d^{3} M^{2}\left\|-\mathbf{E}^{n-1}-\boldsymbol{\Lambda}^{n-1}+\mathbf{u}^{n-1}-\mathbf{u}^{n}\right\|^{2} \\
\leq & \lambda_{M}^{2}\left\|\mathbf{F}^{n}\right\|^{2}+\lambda_{M}^{2} \nu^{2}\left\|\mathbf{F}_{u}^{n}\right\|^{2}+\frac{3}{2} d^{3} M^{2}\left\|\mathbf{E}^{n-1}\right\|^{2}+\frac{3}{2} d^{3} M^{2}\left\|\boldsymbol{\Lambda}^{n-1}\right\|^{2} \\
& +\frac{3}{2} d^{3} M^{2} \Delta t \int_{t^{n-1}}^{t^{n}}\left\|\mathbf{u}_{t}\right\|^{2} d t .
\end{aligned}
$$

In order to estimate the $g_{a}$ terms in $\mathcal{F}_{2}(\cdot)$ note that

$$
\begin{aligned}
\lambda\left(g_{a}\left(\tau_{h}^{n-1}, \nabla \mathbf{u}_{h}^{n-1}\right)-g_{a}\left(\tau^{n}, \nabla \mathbf{u}^{n}\right)\right)=\lambda( & g_{a}\left(\tau_{h}^{n-1}, \nabla\left(\mathbf{u}_{h}^{n-1}-\mathcal{U}^{n-1}\right)\right)+g_{a}\left(\tau_{h}^{n-1}, \nabla\left(\mathcal{U}^{n-1}-\mathbf{u}^{n-1}\right)\right) \\
& +g_{a}\left(\tau_{h}^{n-1}, \nabla\left(\mathbf{u}^{n-1}-\mathbf{u}^{n}\right)\right)+g_{a}\left(\tau_{h}^{n-1}-\mathcal{T}^{n-1}, \nabla \mathbf{u}^{n}\right) \\
& \left.+g_{a}\left(\mathcal{T}^{n-1}-\tau^{n-1}, \nabla \mathbf{u}^{n}\right)+g_{a}\left(\tau^{n-1}-\tau^{n}, \nabla \mathbf{u}^{n}\right)\right) \\
=\quad \lambda( & -g_{a}\left(\tau_{h}^{n-1}, \nabla \mathbf{E}^{n-1}\right)-g_{a}\left(\tau_{h}^{n-1}, \nabla \mathbf{\Lambda}^{n-1}\right) \\
& -g_{a}\left(\tau_{h}^{n-1}, \nabla\left(\mathbf{u}^{n}-\mathbf{u}^{n-1}\right)\right)-g_{a}\left(\mathbf{F}^{n-1}, \nabla \mathbf{u}^{n}\right) \\
& \left.-g_{a}\left(\boldsymbol{\Gamma}^{n-1}, \nabla \mathbf{u}^{n}\right)-g_{a}\left(\tau^{n}-\tau^{n-1}, \nabla \mathbf{u}^{n}\right)\right) .
\end{aligned}
$$

Bounding each of the terms on the right hand side of (4.38) we obtain

$$
\begin{aligned}
& \left|\left(\lambda g_{a}\left(\tau_{h}^{n-1}, \nabla \mathbf{E}^{n-1}\right), \tilde{\mathbf{F}}^{n}\right)\right| \leq\left\|g_{a}\left(\tau_{h}^{n-1}, \nabla \mathbf{E}^{n-1}\right)\right\|\left\|\lambda \tilde{\mathbf{F}}^{n}\right\| \\
& \leq 4 \hat{d}\left\|\tau_{h}^{n-1}\right\|_{\infty}\left\|\nabla \mathbf{E}^{n-1}\right\| \lambda_{M}\left\|\tilde{\mathbf{F}}^{n}\right\| \\
& \leq \epsilon_{8}\left\|\nabla \mathbf{E}^{n-1}\right\|^{2}+\frac{8 \tilde{d}^{2} K^{2} \lambda_{M}^{2}}{\epsilon_{8}}\left\|\mathbf{F}^{n}\right\|^{2}+\frac{8 \tilde{d}^{2} K^{2} \lambda_{M}^{2}}{\epsilon_{8}} \nu^{2}\left\|\mathbf{F}_{u}^{n}\right\|^{2}, \\
& \left|\left(\lambda g_{a}\left(\tau_{h}^{n-1}, \nabla \boldsymbol{\Lambda}^{n-1}\right), \tilde{\mathbf{F}}^{n}\right)\right| \leq 8 \tilde{d}^{2} K^{2}\left\|\nabla \boldsymbol{\Lambda}^{n-1}\right\|^{2}+\lambda_{M}^{2}\left\|\mathbf{F}^{n}\right\|^{2}+\lambda_{M}^{2} \nu^{2}\left\|\mathbf{F}_{u}^{n}\right\|^{2}, \\
& \left|\left(\lambda g_{a}\left(\tau_{h}^{n-1}, \nabla\left(\mathbf{u}^{n}-\mathbf{u}^{n-1}\right)\right), \tilde{\mathbf{F}}^{n}\right)\right| \leq 8 d^{2} K^{2} \Delta t \int_{t^{n-1}}^{t^{n}}\left\|\nabla u_{t}\right\|^{2} d t+\lambda_{M}^{2}\left\|\mathbf{F}^{n}\right\|^{2}+\lambda_{M}^{2} \nu^{2}\left\|\mathbf{F}_{u}^{n}\right\|^{2}, \\
& \left|\left(\lambda g_{a}\left(\mathbf{F}^{n-1}, \nabla \mathbf{u}^{n}\right), \tilde{\mathbf{F}}^{n}\right)\right| \leq 8 \tilde{d}^{2} M^{2}\left\|\mathbf{F}^{n-1}\right\|^{2}+\lambda_{M}^{2}\left\|\mathbf{F}^{n}\right\|^{2}+\lambda_{M}^{2} \nu^{2}\left\|\mathbf{F}_{u}^{n}\right\|^{2}, \\
& \left|\left(\lambda g_{a}\left(\boldsymbol{\Gamma}^{n-1}, \nabla \mathbf{u}^{n}\right), \tilde{\mathbf{F}}^{n}\right)\right| \leq 8 d^{2} M^{2}\left\|\boldsymbol{\Gamma}^{n-1}\right\|^{2}+\lambda_{M}^{2}\left\|\mathbf{F}^{n}\right\|^{2}+\lambda_{M}^{2} \nu^{2}\left\|\mathbf{F}_{u}^{n}\right\|^{2}, \\
& \left|\left(\lambda g_{a}\left(\tau^{n}-\tau^{n-1}, \nabla \mathbf{u}^{n}\right), \tilde{\mathbf{F}}^{n}\right)\right| \leq 8 \dot{d}^{2} M^{2} \Delta t \int_{t^{n-1}}^{t^{n}}\left\|\tau_{t}\right\|^{2} d t+\lambda_{M}^{2}\left\|\mathbf{F}^{n}\right\|^{2}+\lambda_{M}^{2} \nu^{2}\left\|\mathbf{F}_{u}^{n}\right\|^{2} .
\end{aligned}
$$

Combining the estimates in (4.30)-(4.37), (4.39)-(4.44), we obtain the following estimate for $\mathcal{F}_{2}\left(\mathbf{F}^{n}\right)$ :

$$
\begin{aligned}
\left|\mathcal{F}_{2}\left(\mathbf{F}^{n}\right)\right| \leq & \epsilon_{8}\left\|\nabla \mathbf{E}^{n-1}\right\|^{2}+\nu^{2}\left\|\mathbf{F}_{u}^{n}\right\|^{2}\left(6 \lambda_{M}^{2}+3+\frac{8 d^{2} K^{2} \lambda_{M}^{2}}{\epsilon_{8}}\right) \\
& +\left\|\mathbf{F}^{n}\right\|^{2}\left(11 \lambda_{M}^{2}+2+\frac{8 \dot{d}^{2} K^{2} \lambda_{M}^{2}}{\epsilon_{8}}+\dot{d}\left\|\nabla \mathbf{E}^{n-1}\right\|_{\infty}\right)
\end{aligned}
$$




$$
\begin{aligned}
& +\left\|\mathbf{E}^{n-1}\right\|^{2}\left(\frac{3}{2} \dot{d}^{3} M^{2}\right)+\left\|\mathbf{F}^{n-1}\right\|^{2}\left(8 \dot{d}^{2} M^{2}\right) \\
& +2 \alpha^{2}\left\|\nabla \boldsymbol{\Lambda}^{n}\right\|^{2}+\left\|\nabla \boldsymbol{\Gamma}^{n}\right\|^{2}\left(\frac{\dot{d} K^{2}}{2}\right)+\left\|\boldsymbol{\Gamma}^{n}\right\|^{2}\left(\frac{1}{2}\right)+\left\|d_{t} \boldsymbol{\Gamma}^{n}\right\|^{2}\left(\frac{1}{4}\right) \\
& +\left\|\nabla \boldsymbol{\Lambda}^{n-1}\right\|^{2}\left(8 \dot{d}^{2} K^{2}\right)+\left\|\boldsymbol{\Lambda}^{n-1}\right\|^{2}\left(\frac{3}{2} \dot{d}^{3} M^{2}\right)+\left\|\boldsymbol{\Gamma}^{n-1}\right\|^{2}\left(8 \dot{d}^{2} M^{2}\right) \\
& +\frac{1}{4}\left\|d_{t} \tau^{n}-\tau_{t}^{n}\right\|^{2}+\frac{\nu^{2}}{4}\left(\hat{d}^{2} M^{2}+\hat{d}\left\|\nabla \mathbf{E}^{n-1}\right\|_{\infty}\right)\left\|\tau_{t}^{n}\right\|^{2}+\frac{\nu^{2}}{4} K^{2} \dot{d}\left\|\nabla \tau_{t}^{n}\right\|^{2} \\
& +\frac{3}{2} \hat{d}^{3} M^{2} \Delta t \int_{t^{n-1}}^{t^{n}}\left\|\mathbf{u}_{t}\right\|^{2} d t+8 \dot{d}^{2} M^{2} \Delta t \int_{t^{n-1}}^{t^{n}}\left\|\tau_{t}\right\|^{2} d t+8 \dot{d}^{2} K^{2} \Delta t \int_{t^{n-1}}^{t^{n}}\left\|\nabla \mathbf{u}_{t}\right\|^{2} d t .
\end{aligned}
$$

Then, from (4.18) we have that

$$
\begin{aligned}
& \alpha R e_{m}\left(\left\|\mathbf{E}^{l}\right\|^{2}-\left\|\mathbf{E}^{0}\right\|^{2}\right)+\frac{\lambda_{m}}{2}\left(\left\|\mathbf{F}^{l}\right\|^{2}-\left\|\mathbf{F}^{0}\right\|^{2}\right)+4 \alpha(1-\alpha) \sum_{n=1}^{l} \frac{R e_{m} \Delta t}{R e_{M}}\left\|D\left(\mathbf{E}^{n}\right)\right\|^{2}+\nu \sum_{n=1}^{l} \frac{\lambda_{m}^{2} \Delta t}{\lambda_{M}}\left\|\mathbf{F}_{u}^{n}\right\|^{2} \\
& +\sum_{n=1}^{l} \frac{\lambda_{m} \Delta t}{\lambda_{M}}\left\|\mathbf{F}^{n}\right\|^{2} \leq \Delta t \sum_{n=1}^{l}\left\{4 \alpha^{2}\left(C_{K}^{2} R e_{M}^{2} \epsilon_{1}+\tilde{R}^{2} \epsilon_{2}+\epsilon_{3}\right)+2 \alpha\left(C_{K}^{2} \epsilon_{5}+(1-\alpha) \epsilon_{6}+\epsilon_{7}\right)\right\}\left\|D\left(\mathbf{E}^{n}\right)\right\|^{2} \\
& +\Delta t \sum_{n=1}^{l} C_{K}^{2} \epsilon_{8}\left\|D\left(\mathbf{E}^{n-1}\right)\right\|^{2}+\Delta t \sum_{n=1}^{l}\left(\frac{\dot{d} K^{2}}{4 \epsilon_{1}}+2 \alpha\left(3 R e_{M}^{2}+\frac{3}{2}\right)\right)\left\|\mathbf{E}^{n}\right\|^{2} \\
& +\Delta t \sum_{n=1}^{l}\left(\frac{3 \dot{d}^{2} M^{2}}{2}+\alpha R e_{M}^{2} \hat{d}^{2} M^{2}\right)\left\|\mathbf{E}^{n-1}\right\|^{2} \\
& +\Delta t \sum_{n=1}^{l}\left\{\left(\frac{\lambda_{M}}{2}+1\right) \dot{d}\left\|\nabla \mathbf{E}^{n-1}\right\|_{\infty}+\frac{\lambda_{M} \dot{d} M}{2}+\frac{1}{4 \epsilon_{2}}+3+11 \lambda_{M}^{2}+\frac{8 \dot{d}^{2} K^{2} \lambda_{M}^{2}}{\epsilon_{8}}\right\}\left\|\mathbf{F}^{n}\right\|^{2} \\
& +\Delta t \sum_{n=1}^{l} 8 \tilde{d}^{2} M^{2}\left\|\mathbf{F}^{n-1}\right\|^{2} \\
& +\Delta t \sum_{n=1}^{l} \nu^{2}\left(\frac{1}{4 \epsilon_{3}}+\frac{1}{4}+6 \lambda_{M}^{2}+3+\frac{8 d^{2} K^{2} \lambda_{M}^{2}}{\epsilon_{8}}\right)\left\|\mathbf{F}_{u}^{n}\right\|^{2} \\
& +\Delta t \sum_{n=1}^{l} \frac{2 \alpha \dot{d}}{4 \epsilon_{5}}\left\|p^{n}-\mathbf{P}^{n}\right\|^{2}+\Delta t \sum_{n=1}^{l}\left(\alpha R e_{M}^{2} \dot{d}^{2} M^{2}+\frac{3 \dot{d}^{3} M^{2}}{2}\right)\left\|\boldsymbol{\Lambda}^{n-1}\right\|^{2} \\
& +\Delta t \sum_{n=1}^{l} 2 \alpha\left(\frac{K^{2} \dot{d}}{4}+\frac{1-\alpha}{\epsilon_{6}}+2 \alpha^{2}\right)\left\|\nabla \boldsymbol{\Lambda}^{n}\right\|^{2}+\Delta t \sum_{n=1}^{l} 8 \dot{d}^{2} K^{2}\left\|\nabla \boldsymbol{\Lambda}^{n-1}\right\|^{2} \\
& +\Delta t \sum_{n=1}^{l} \frac{\alpha}{2}\left\|d_{t} \boldsymbol{\Lambda}^{n}\right\|^{2}+\Delta t \sum_{n=1}^{l} \frac{1}{4}\left\|d_{t} \boldsymbol{\Gamma}^{n}\right\|^{2}+\Delta t \sum_{n=1}^{l}\left(\frac{\alpha}{2 \epsilon_{7}}+\frac{1}{2}\right)\left\|\boldsymbol{\Gamma}^{n}\right\|^{2} \\
& +\Delta t \sum_{n=1}^{l} \frac{d K^{2}}{2}\left\|\nabla \boldsymbol{\Gamma}^{n}\right\|^{2}+\Delta t \sum_{n=1}^{l} 8 \dot{d}^{2} M^{2}\left\|\boldsymbol{\Gamma}^{n-1}\right\|^{2} \\
& +\Delta t \sum_{n=1}^{l} \frac{\alpha}{2}\left\|d_{t} \mathbf{u}^{n}-\mathbf{u}_{t}^{n}\right\|^{2}+\Delta t \sum_{n=1}^{l} \frac{1}{4}\left\|d_{t} \tau^{n}-\tau_{t}^{n}\right\|^{2} \\
& +\Delta t \sum_{n=1}^{l} \frac{\nu^{2}}{4}\left(\dot{d}^{2} M^{2}+\dot{d}\left\|\nabla \mathbf{E}^{n-1}\right\|_{\infty}\right)\left\|\tau_{t}^{n}\right\|^{2}
\end{aligned}
$$




$$
\begin{aligned}
& +\Delta t \sum_{n=1}^{l}\left(\alpha R e_{M}^{2} \dot{d}^{2} M^{2} \Delta t+\frac{3}{2} d^{3} M^{2} \Delta t\right) \int_{t^{n-1}}^{t^{n}}\left\|\mathbf{u}_{t}\right\|^{2} d t \\
& +\Delta t \sum_{n=1}^{l} \frac{\nu^{2}}{4} K^{2} \dot{d}\left\|\nabla \tau_{t}^{n}\right\|^{2}+\Delta t \sum_{n=1}^{l} 8 \dot{d}^{2} M^{2} \Delta t \int_{t^{n-1}}^{t^{n}}\left\|\tau_{t}\right\|^{2} d t \\
& +\Delta t \sum_{n=1}^{l} 8 \dot{d}^{2} K^{2} \Delta t \int_{t^{n-1}}^{t^{n}}\left\|\nabla \mathbf{u}_{t}\right\|^{2} d t .
\end{aligned}
$$

With the following choices:

$$
\begin{array}{ccc}
\epsilon_{1}=\frac{R e_{m}(1-\alpha)}{14 C_{K}^{2} \operatorname{Re}_{M}^{3} \alpha}, & \epsilon_{2}=\frac{R e_{m}(1-\alpha)}{14 \tilde{R}^{2} R e_{M} \alpha}, & \epsilon_{3}=\frac{R e_{m}(1-\alpha)}{14 R e_{M} \alpha}, \\
\epsilon_{5}=\frac{R e_{m}(1-\alpha)}{7 C_{K}^{2} R e_{M}}, & \epsilon_{6}=\frac{R e_{m}}{7 R e_{M}}, & \epsilon_{7}=\frac{R e_{m}(1-\alpha)}{7 R e_{M}}, \\
\epsilon_{8}=\frac{R e_{m} 2 \alpha(1-\alpha)}{7 \operatorname{Re}_{M} C_{K}^{2}}, & \mathbf{u}_{h}^{0}=\mathcal{U}^{0}\left(\Rightarrow \mathbf{E}^{0}=\mathbf{0}\right), & \tau_{h}^{0}=\mathcal{T}^{0}\left(\Rightarrow \mathbf{F}^{0}=\mathbf{0}\right),
\end{array}
$$

substituting into (4.46) yields

$$
\begin{aligned}
\alpha R e_{m}\left\|\mathbf{E}^{l}\right\|^{2}+\frac{\lambda_{m}}{2}\left\|\mathbf{F}^{l}\right\|^{2} & +2 \alpha(1-\alpha) \frac{R e_{m}}{R e_{M}} \Delta t \sum_{n=1}^{l}\left\|D\left(\mathbf{E}^{n}\right)\right\|^{2} \\
+ & \left(\nu \frac{\lambda_{m}^{2}}{\lambda_{M}}-\nu^{2}\left(\frac{7 \tilde{R}^{2} R e_{M} \alpha}{2 R e_{m}(1-\alpha)}+\frac{28 \dot{d}^{2} C_{K}^{2} K^{2} \lambda_{M}^{2} R e_{M}}{\alpha(1-\alpha) R e_{m}}+\frac{13}{4}+6 \lambda_{M}^{2}\right)\right) \Delta t \sum_{n=1}^{l}\left\|\mathbf{F}_{u}^{n}\right\|^{2} \\
+\frac{\lambda_{m}}{\lambda_{M}} \Delta t \sum_{n=1}^{l}\left\|\mathbf{F}^{n}\right\|^{2} \leq & C_{1} \Delta t \sum_{n=1}^{l}\left\|\mathbf{E}^{n}\right\|^{2}+\Delta t \sum_{n=1}^{l}\left(C_{2}\left(1+\left\|\nabla \mathbf{E}^{n-1}\right\|_{\infty}\right)\right)\left\|\mathbf{F}^{n}\right\|^{2} \\
& +C_{3} \Delta t \sum_{n=1}^{l}\left\|\mathbf{\Lambda}^{n}\right\|^{2}+C_{4} \Delta t \sum_{n=1}^{l}\left\|\nabla \boldsymbol{\Lambda}^{n}\right\|^{2}+\frac{\alpha}{2} \Delta t \sum_{n=1}^{l}\left\|d_{t} \boldsymbol{\Lambda}^{n}\right\|^{2} \\
& +\frac{1}{4} \Delta t \sum_{n=1}^{l}\left\|d_{t} \boldsymbol{\Gamma}^{n}\right\|^{2}+C_{5} \Delta t \sum_{n=1}^{l}\left\|\boldsymbol{\Gamma}^{n}\right\|^{2}+C_{6} \Delta t \sum_{n=1}^{l}\left\|\nabla \boldsymbol{\Gamma}^{n}\right\|^{2} \\
& +\frac{\alpha}{2} \Delta t \sum_{n=1}^{l}\left\|d_{t} \mathbf{u}^{n}-\mathbf{u}_{t}^{n}\right\|^{2}+\frac{1}{4} \Delta t \sum_{n=1}^{l}\left\|d_{t} \tau^{n}-\tau_{t}^{n}\right\|^{2} \\
& +\frac{2 \alpha \dot{d}}{4 \epsilon_{5}} \Delta t \sum_{n=1}^{l}\left\|p^{n}-\mathbf{P}^{n}\right\|^{2} \\
& +\Delta t \sum_{n=1}^{l} \frac{\nu^{2}}{4}\left(\dot{d}^{2} M^{2}+\dot{d}\left\|\nabla \mathbf{E}^{n-1}\right\| \|_{\infty}\right)\left\|\tau_{t}^{n}\right\|^{2} \\
& +|\Delta t|^{2}\left(\alpha R e_{M}^{2} \dot{d}^{2} M^{2}+\frac{3}{2} \dot{d}^{3} M^{2}\right)\left\|\mathbf{u}_{t}\right\|_{0,0}^{2}+K^{2} \dot{d} \frac{\nu^{2}}{4}\left\|\nabla \tau_{t}\right\|_{0,0}^{2} \\
& +8 \dot{d}^{2} M^{2}|\Delta t|^{2}\left\|\tau_{t}\right\|_{0,0}^{2}+8 \dot{d}^{2} K^{2}|\Delta t|^{2}\left\|\nabla \mathbf{u}_{t}\right\|_{0,1}^{2} .
\end{aligned}
$$

We now apply the interpolation properties of the approximating spaces to estimate the terms on the right hand side of (4.47). Using elements of order $k$ for velocity, elements of order $m$ for stress, 
and elements of order $q$ for pressure, we have

$$
\begin{gathered}
\sum_{n=1}^{l} \Delta t\left\|\nabla \boldsymbol{\Lambda}^{n}\right\|^{2}+\sum_{n=1}^{l} \Delta t\left\|\nabla \boldsymbol{\Gamma}^{n}\right\|^{2} \leq C\left(h^{2 k} \sum_{n=1}^{l} \Delta t\left\|\mathbf{u}^{n}\right\|_{k+1}^{2}+h^{2 m} \sum_{n=1}^{l} \Delta t\left\|\tau^{n}\right\|_{m+1}^{2}\right) \\
\leq C\left(h^{2 k}\|\mathbf{u}\|_{0, k+1}^{2}+h^{2 m}\|\tau\|_{0, m+1}^{2}\right) \\
\sum_{n=1}^{l} \Delta t\left\|\boldsymbol{\Lambda}^{n}\right\|^{2}+\sum_{n=1}^{l} \Delta t\left\|\boldsymbol{\Gamma}^{n}\right\|^{2}+\sum_{n=1}^{l} \Delta t\left\|p-\mathcal{P}^{n}\right\|^{2} \\
\leq C\left(h^{2 k+2} \sum_{n=1}^{l} \Delta t\left\|\mathbf{u}^{n}\right\|_{k+1}^{2}+h^{2 m+2} \sum_{n=1}^{l} \Delta t\left\|\tau^{n}\right\|_{m+1}^{2}+h^{2 q+2} \sum_{n=1}^{l} \Delta t\left\|p^{n}\right\|_{q+1}^{2}\right) \\
\leq C\left(h^{2 k+2}\|\mathbf{u}\|_{0, k+1}^{2}+h^{2 m+2}\|\mid \tau\|_{0, m+1}^{2}+h^{2 q+2}\|\|_{0, q+1}^{2}\right), \\
\quad=\sum_{n=1}^{l} \Delta t\left\|\frac{1}{\Delta t} \int_{t_{n-1}}^{t_{n}} 1 \frac{\partial \Lambda}{\partial t} d t\right\|^{2} \\
\leq \sum_{n=1}^{l} \Delta t\left(\frac{1}{\Delta t}\right)^{2} \int_{\Omega}\left(\int_{t_{n-1}}^{t_{n}} 1 d t\right)\left(\int_{t_{n-1}}^{t_{n}}\left(\frac{\partial \Lambda}{\partial t}\right)^{2} d t\right) d \mathbf{x} \\
\leq C^{2 k+2}\left\|\mathbf{u}_{t}\right\|_{0, k+1}^{2},
\end{gathered}
$$

and similarly,

$$
\sum_{n=1}^{l} \Delta t\left\|d_{t} \boldsymbol{\Gamma}^{n}\right\|^{2} \leq C h^{2 m+2}\left\|\tau_{t}\right\|_{0, m+1}^{2} .
$$

Note that $d_{t} \mathbf{u}^{n}-\mathbf{u}_{t}^{n}$ may be expressed as

$$
d_{t} \mathbf{u}^{n}-\mathbf{u}_{t}^{n}=\frac{1}{2 \Delta t} \int_{t_{n-1}}^{t_{n}} \mathbf{u}_{t t}(\cdot, t)\left(t_{n-1}-t\right) d t .
$$

Also,

$$
\begin{aligned}
\left(\frac{1}{2 \Delta t} \int_{t_{n-1}}^{t_{n}} \mathbf{u}_{t t}(\cdot, t)\left(t_{n-1}-t\right) d t\right)^{2} & \leq \frac{1}{4|\Delta t|^{2}} \int_{t_{n-1}}^{t_{n}} \mathbf{u}_{t t}(\cdot, t)^{2} d t \int_{t_{n-1}}^{t_{n}}\left(t_{n-1}-t\right)^{2} d t \\
& =\frac{1}{12} \Delta t \int_{t_{n-1}}^{t_{n}} \mathbf{u}_{t t}^{2}(\cdot, t) d t
\end{aligned}
$$

Therefore it follows that

$$
\begin{aligned}
\sum_{n=1}^{l} \Delta t\left\|d_{t} \mathbf{u}^{n}-\mathbf{u}_{t}^{n}\right\|^{2} & \leq \sum_{n=1}^{l} \Delta t \int_{\Omega} \frac{1}{12} \Delta t \int_{t_{n-1}}^{t_{n}} \mathbf{u}_{t t}^{2}(\cdot, t) d t d \mathbf{x} \\
& =\frac{1}{12}|\Delta t|^{2}\left\|\mathbf{u}_{t t}\right\|_{0,0}^{2} .
\end{aligned}
$$

Similarly, for $d_{t} \tau^{n}-\tau_{t}^{n}$ we have

$$
\sum_{n=1}^{l} \Delta t\left\|d_{t} \tau^{n}-\tau_{t}^{n}\right\|^{2} \leq \frac{1}{12}|\Delta t|^{2}\left\|\tau_{t t}\right\|_{0,0}^{2} .
$$


In view of (4.48)-(4.53), our induction hypotheses (IH1),(IH2), and with $\nu$ chosen such that

$$
\nu \leq \frac{1}{2} \frac{\lambda_{m}^{2}}{\lambda_{M}}\left(\frac{7 \tilde{R}^{2} R e_{M} \alpha}{2 \operatorname{Re}_{m}(1-\alpha)}+\frac{28 \tilde{d}^{2} C_{K}^{2} K^{2} \lambda_{M}^{2} R e_{M}}{\alpha(1-\alpha) R e_{m}}+\frac{13}{4}+6 \lambda_{M}^{2}\right)^{-1}
$$

from (4.47) we obtain

$$
\begin{aligned}
\alpha R e_{m} & \left\|\mathbf{E}^{l}\right\|^{2}+\frac{\lambda_{m}}{2}\left\|\mathbf{F}^{l}\right\|^{2}+2 \alpha(1-\alpha) \frac{R e_{m}}{R e_{M}} \Delta t \sum_{n=1}^{l}\left\|D\left(\mathbf{E}^{n}\right)\right\|^{2}+\frac{\nu}{2} \frac{\lambda_{m}^{2}}{\lambda_{M}} \sum_{n=1}^{l} \Delta t\left\|\mathbf{F}_{u}^{n}\right\|^{2} \\
\leq & C \sum_{n=1}^{l} \Delta t\left(\left\|\mathbf{E}^{n}\right\|^{2}+\left\|\mathbf{F}^{n}\right\|^{2}\right)+C \sum_{n=1}^{l} \Delta t\left\|\nabla \mathbf{E}^{n-1}\right\|_{\infty}\left\|\mathbf{F}^{n}\right\|^{2}+\nu^{2} C\left\|\tau_{t}\right\|_{0,1}^{2} \\
& +\frac{\nu^{2}}{4} \sum_{n=1}^{l} \Delta t\left\|\nabla \mathbf{E}^{n-1}\right\|_{\infty}\left\|\tau_{t}^{n}\right\|^{2}+C|\Delta t|^{2}\left(\left\|\mathbf{u}_{t}\right\|_{0,1}^{2}+\left\|\tau_{t}\right\|_{0,0}^{2}+\left\|\mathbf{u}_{t t}\right\|_{0,0}^{2}+\left\|\tau_{t t}\right\|_{0,0}^{2}\right) \\
& +C h^{2 k+2}\|\mathbf{u}\|_{0, k+1}^{2}+C h^{2 m+2}\|\tau\|_{0, m+1}^{2}+C h^{2 q+2}\|p\|_{0, q+1}^{2}+C h^{2 k}\|\mathbf{u}\|_{0, k+1}^{2} \\
& +C h^{2 k+2}\left\|\mathbf{u}_{t}\right\|_{0, k+1}^{2}+C h^{2 m}\|\tau\|_{0, m+1}^{2}+C h^{2 m+2}\left\|\tau_{t}\right\|_{0, m+1}^{2},
\end{aligned}
$$

where the $C^{\prime} s$ denote constants independent of $l, \Delta t, h, \nu$. Applying Gronwall's lemma and (IH2) to (4.55), the estimate given in (4.7) follows.

Step 2. We show that the induction hypotheses, $(I H 1)$ and $(I H 2)$ are true.

\section{Verification of (IH1)}

Assume that $(I H 1)$ holds true for $n=1,2, \ldots, l-1$. By interpolation properties, inverse estimates and (4.7), we have that

$$
\begin{aligned}
\left\|\mathbf{u}_{h}^{l}\right\|_{\infty} & \leq\left\|\mathbf{u}_{h}^{l}-\mathbf{u}^{l}\right\|_{\infty}+\left\|\mathbf{u}^{l}\right\|_{\infty} \\
& \leq\left\|\mathbf{E}^{l}\right\|_{\infty}+\left\|\Lambda^{l}\right\|_{\infty}+M \\
& \leq C h^{-\frac{\dot{d}}{2}}\left\|\mathbf{E}^{l}\right\|_{0}+C h^{-\frac{\dot{d}}{2}}\left\|\Lambda^{l}\right\|_{0}+M \\
& \leq C\left(|\Delta t| h^{-\frac{\dot{d}}{2}}+\nu h^{-\frac{\dot{d}}{2}}+h^{k-\frac{\dot{d}}{2}}+h^{m-\frac{\dot{d}}{2}}+h^{q+1-\frac{\dot{d}}{2}}+h^{k+1-\frac{\dot{d}}{2}}\right)+M .
\end{aligned}
$$

Note that the expression $C\left(|\Delta t| h^{-\frac{\dot{d}}{2}}+\nu h^{-\frac{\dot{d}}{2}}+h^{k-\frac{\dot{d}}{2}}+h^{m-\frac{\dot{d}}{2}}+h^{q+1-\frac{\dot{d}}{2}}+h^{k+1-\frac{\hat{d}}{2}}\right)$ is independent of $l$. Hence, if we set $k, m \geq \frac{\dot{d}}{2}, q \geq \frac{\dot{d}}{2}-1$, and choose $h, \Delta t, \nu$ such that

$$
h^{k-\frac{\dot{d}}{2}}, h^{m-\frac{\dot{d}}{2}}, h^{q+1-\frac{\dot{d}}{2}} \leq \frac{1}{C}, \quad \Delta t, \nu \leq \frac{h^{\frac{d}{2}}}{C},
$$

then from (4.56)

$$
\left\|\mathbf{u}_{h}^{l}\right\|_{\infty} \leq M+6 .
$$

Similarly it follows that $\left\|\tau_{h}^{l}\right\|_{\infty} \leq M+6$. 


\section{Verification of $(\mathrm{IH} 2)$}

Assume that $(I H 2)$ is true for $n=1,2, \ldots, l-1$. Equations (4.7), (4.55), and Korn's inequality imply

$$
\sum_{n=1}^{l} \Delta t\left\|\nabla \mathbf{E}^{n}\right\|_{0}^{2} \leq C\left(h^{2 k}+h^{2 m}+h^{2 q+2}+|\Delta t|^{2}+\nu^{2}\right) .
$$

Applying the inverse estimate and using the inequality

$$
\sum_{n=1}^{l} a_{n} \leq \sqrt{l}\left(\sum_{n=1}^{l} a_{n}^{2}\right)^{\frac{1}{2}}
$$

from (4.58) we obtain

$$
\begin{aligned}
\sum_{n=1}^{l} \Delta t\left\|\nabla \mathbf{E}^{n}\right\|_{\infty} & \leq C h^{-\frac{\dot{d}}{2}} \sum_{n=1}^{l} \Delta t\left\|\nabla \mathbf{E}^{n}\right\| \\
& \leq C h^{-\frac{\dot{d}}{2}} \sqrt{\Delta} t \sqrt{l}\left(\sum_{n=1}^{l} \Delta t\left\|\nabla \mathbf{E}^{n}\right\|^{2}\right)^{\frac{1}{2}} \\
& \leq \tilde{C}\left(\Delta t h^{-\frac{\dot{d}}{2}}+\nu h^{-\frac{\dot{d}}{2}}+h^{k-\frac{\dot{d}}{2}}+h^{m-\frac{\dot{d}}{2}}+h^{q+1-\frac{\dot{d}}{2}}\right),
\end{aligned}
$$

where $\tilde{C}=C \sqrt{T}$ is a constant independent of $l, h, \Delta t$, and $\nu$. Hence when

$$
\nu, \Delta t \leq \frac{h^{\frac{d}{2}}}{5 \tilde{C}}
$$

and

( IH2) holds.

$$
h^{k-\frac{d}{2}}, h^{m-\frac{d}{2}}, h^{q+1-\frac{d}{2}} \leq \frac{1}{5 \tilde{C}},
$$

Step 3. We derive the error estimates in (4.2) and (4.3).

Proof of the Theorem 4.1.

Using estimates (4.7) and (approximation properties), we have

$$
\begin{aligned}
\left\|\mathbf{u}-\mathbf{u}_{h}\right\|_{\infty, 0}^{2}+\left\|\tau-\tau_{h}\right\|_{\infty, 0}^{2} & \leq\|\mathbf{E}\|_{\infty, 0}^{2}+\|\Lambda\|_{\infty, 0}^{2}+\|\mathbf{F}\|_{\infty, 0}^{2}+\|\Gamma\|_{\infty, 0}^{2} \\
& \leq G(\Delta t, h, \nu)+C\left(h^{2 k+2}\|\mathbf{u}\|_{\infty, k+1}^{2}+h^{2 m+2}\|\tau\|_{\infty, m+1}^{2}\right) .
\end{aligned}
$$

Note the restrictions on $\nu$ and $\Delta t$ from (4.54), (4.57), (4.59), (3.21), (3.22), and the hypothesis of Theorem 4.1.

To establish (4.3), from (4.7), (4.55), we have

$$
\|\nabla \mathbf{E}\|_{0,0}^{2} \leq C(T+1) G(\Delta t, h, \nu)
$$

and

$$
\|\mathbf{E}\|_{0,0}^{2}+\|\mathbf{F}\|_{0,0}^{2} \leq T G(\Delta t, h, \nu) .
$$

Hence

$$
\|\mathbf{E}\|_{1,0}^{2}+\|\mathbf{F}\|_{0,0}^{2} \leq \tilde{C} G(\Delta t, h, \nu) .
$$




\section{$5 \quad$ Numerical Results}

In this section, we present two numerical simulations of viscoelastic fluid flow involving two immiscible fluids. For a discussion on the numerical implementation of the continuum surface force model see $[22]$.

\section{Example 1: An Elliptical Minor Phase Evolving to a Circular Shape}

Let $\Omega:=(0,1) \times(0,1)$, and at $t=0$, let

$$
\Omega_{1}:=\left\{(x, y): \frac{(x-0.5)^{2}}{0.35^{2}}+\frac{y-0.5)^{2}}{0.25^{2}}<1\right\}, \mathcal{I}:=\left\{(x, y): \frac{(x-0.5)^{2}}{0.35^{2}}+\frac{y-0.5)^{2}}{0.25^{2}}=1\right\}
$$

and $\Omega_{2}=\Omega \backslash\left(\Omega_{1} \cup \mathcal{I}\right)$. Initially, both fluids are at rest, $\mathbf{u}(\mathbf{x}, 0)=\mathbf{0}$. We assume, $R e_{1}=R e_{2}=1.0$ and $\lambda_{1}=\lambda_{2}=0.1$. It is common in polymer processing that two fluids have very similar properties so the above assumptions are reasonable. Also, the coefficient of interfacial tension is assumed to be constant, $\sigma=5.0$.

From a minimum energy argument, we have that the interfacial forces will drive $\Omega_{1}$ from its initial elliptical profile to a circular orientation.

In the computations we use for $\nu$, the SUPG coeffecient, $\nu=0.6 h$, and take $\Delta t=h / 2$. To approximate the velocity and pressure we use the Taylor-Hood approximation pair (continuous piecewise quadratics for velocity, continuous piecewise linears for pressure) and use a continuous piecewise linear approximation for the polymeric stress.

Presented in Figures 5.1, 5.2, 5.3, and 5.4 is the velocity field and the interface $\mathcal{I}$ at times $t=$ $0.00,0.11,0.55$, and 3.54 , for the grid with $h=1 / 64$.

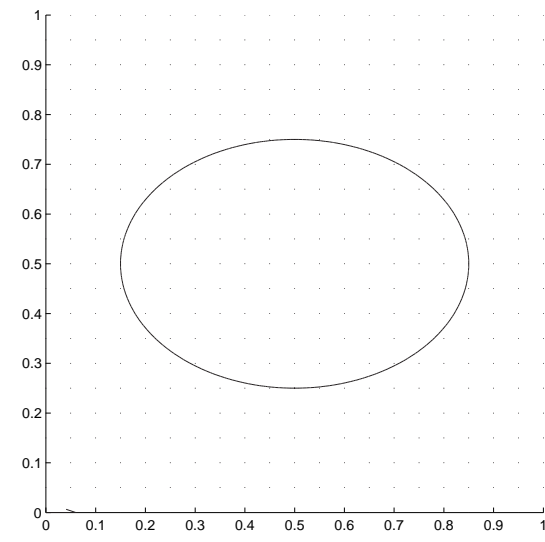

Figure 5.1: Initial velocity field

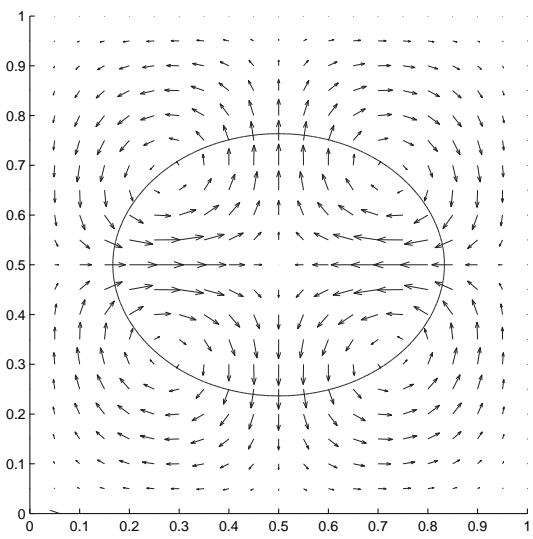

Figure 5.2: Velocity field after 10 time steps 


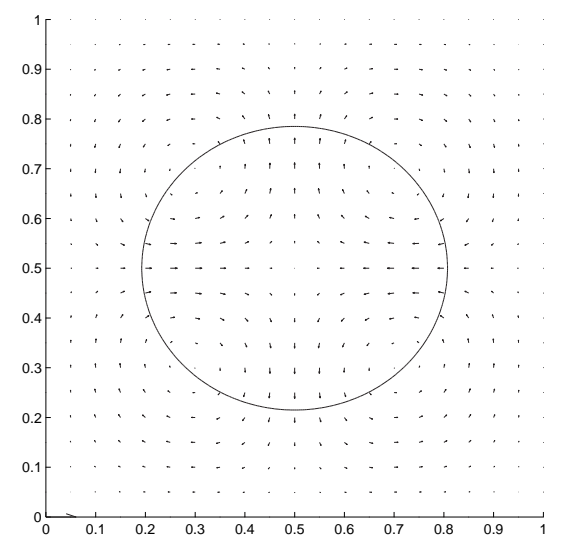

Figure 5.3: Velocity field after 50 time steps

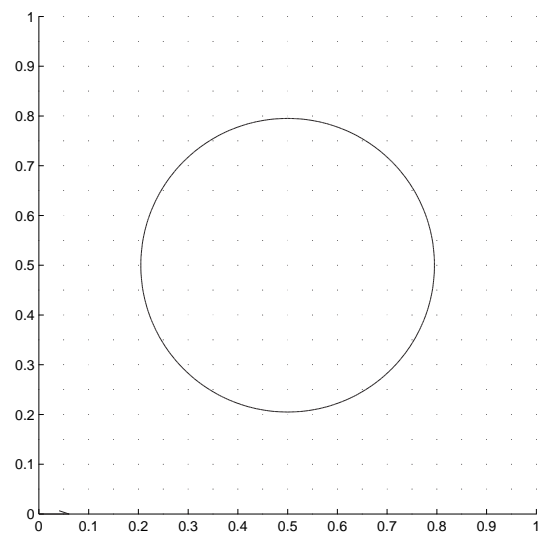

Figure 5.4: Velocity field after 320 time steps

In Table 5.1, we list $\left\|\mathbf{u}_{h} \mid\right\|_{0,1}$ and $\left\|\tau_{h}\right\|_{0,0}$ at time $T=3.536$, together with their experimental convergence rates. The experimental convergence rate for $\left\|\mathbf{u}_{h}\right\|_{0,1}$ was computed as follows. From Theorem 4.1, the choice of approximating elements used, $\nu=0.6 h$, and $\Delta t=h / 2$, we have

$$
\left\|\mathbf{u}_{h}\left|\left\|_{0,1}-\right\| \mathbf{u}\right|\right\|_{0,1} \leq\left\|\mathbf{u}_{h}-\mathbf{u}\right\|_{0,1} \leq C_{v} h .
$$

Using $\left\|\mathbf{u}_{1 / 64} \mid\right\|_{0,1}-\|\mathbf{u}\|_{0,1}=C \frac{1}{64}$ and $\left\|\mathbf{u}_{1 / 48}\right\|_{0,1}-\|\mathbf{u}\|_{0,1}=C \frac{1}{48}$, we obtain an estimate for $\|\mathbf{u}\|_{0,1} \sim\left\|\mathbf{u}_{\infty} \mid\right\|=0.455327$ and an estimate for $C_{v}=1.072320$.

Using $\left\|\mathbf{u}_{\infty}\right\|_{0,1}$ we then compute the experimental convergence rates for $\left\|\mathbf{u}_{h}\right\|_{0,1}$ given in Table 5.1. The experimental convergence rates for $\left\|\tau_{h}\right\|_{0,0}$ are computed analogously. From Theorem 4.1 and (5.63), we have that the theoretical asymptotic convergence rates for $\left\|\left|\mathbf{u}_{h}\right|\right\|_{0,1}$ and $\left\|\tau_{h} \mid\right\|_{0,0}$ is 1 .

\begin{tabular}{|l|l|l|l|l|}
\hline \hline$h$ & $\left\|\mathbf{u}_{h}\right\|_{0,1}$ & $\begin{array}{l}\text { Exp. conv. } \\
\text { rate }\end{array}$ & $\left\|\tau_{h}\right\|_{0,0}$ & $\begin{array}{l}\text { Exp. conv. } \\
\text { rate }\end{array}$ \\
\hline $1 / 32$ & .422046 & 1.00 & .463853 & 1.00 \\
\hline $1 / 40$ & .428505 & 1.00 & .458594 & 1.00 \\
\hline $1 / 45$ & .431560 & 1.00 & .456062 & 1.00 \\
\hline $1 / 48$ & .432987 & 1.00 & .454650 & 1.00 \\
\hline $1 / 50$ & .434386 & 1.01 & .454567 & 0.99 \\
\hline $1 / 54$ & .436445 & 1.01 & .453620 & 0.98 \\
\hline $1 / 64$ & .438572 & 1.00 & .449975 & 1.00 \\
\hline \hline$\infty$ & .455327 & & .435950 & \multicolumn{1}{|l}{} \\
\hline
\end{tabular}

Table 5.1: Experimental Rates of Convergence for Example 1

\section{Example 2: Dual Movement Circular Cavity}

In this example, the inner rod of a circular cavity moves in a counter clockwise direction while the outer wall of the cavity moves in a clockwise direction simultaneously. Both inner and outer velocities have magnitude of one. Figures 5.5 and 5.6 give an indication of the role that interfacial 
tension can play in the mixing process. Initially, the region of minor phase fluid is circular, with radius 0.2 and center $(0,-.67)$. Figures 5.5 and 5.6 present the interfacial shape at time $t=1.5$ for $\sigma=0.3$ and $\sigma=1.0$, respectively. The following parameters are used in the simulation:

$$
R e_{1}=R e_{2}=1.0, \lambda_{1}=\lambda_{2}=0.1, h=0.0590808, \text { and } \Delta t=\frac{h}{2} .
$$

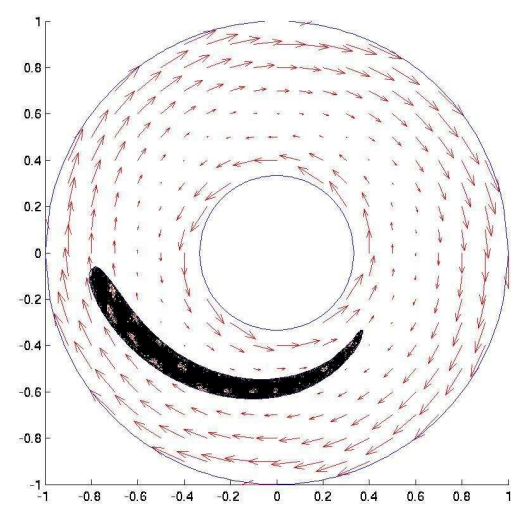

Figure 5.5: Interface after 140 time steps: $\sigma=0.3$

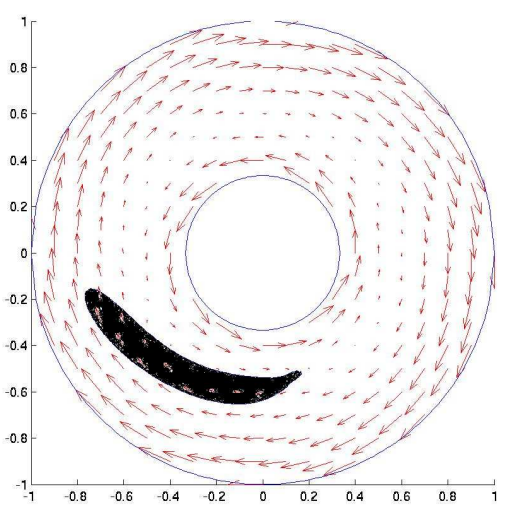

Figure 5.6: Interface after 150 time steps: $\sigma=1.0$

Experimental convergence rates for the case $\sigma=0.3$ are presented in Table 5.2.

\begin{tabular}{|l|l|l|l|l|}
\hline \hline$h$ & $\left\|\mathbf{u}_{h} \mid\right\|_{0,1}$ & $\begin{array}{l}\text { Exp. conv. } \\
\text { rate }\end{array}$ & $\left\|\tau_{h}\right\|_{0,0}$ & $\begin{array}{l}\text { Exp. conv. } \\
\text { rate }\end{array}$ \\
\hline $1 / 21$ & 7.38302 & 1.00 & 2.64138 & 1.00 \\
\hline $1 / 24$ & 7.37221 & 1.00 & 2.63755 & 1.00 \\
\hline $1 / 27$ & 7.36414 & 1.00 & 2.63481 & 1.00 \\
\hline $1 / 30$ & 7.35769 & 1.00 & 2.63243 & 1.00 \\
\hline $1 / 33$ & 7.35256 & 1.00 & 2.63052 & 1.00 \\
\hline$\infty$ & 7.29958 & & 2.61289 & \\
\hline
\end{tabular}

Table 5.2: Experimental Rates of Convergence for Example 2: $\sigma=0.3$ 


\section{References}

[1] J. Baranger and A. Machmoum. Existence of approximate solutions and error bounds for viscoelastic fluid flow: Characteristics method. Comput. Methods Appl. Mech. Engrg., 148:39$52,1997$.

[2] J. Baranger and D. Sandri. Finite element approximation of viscoelastic fluid flow: Existence of approximate solutions and error bounds. I. Discontinuous constraints. Numer. Math., 63:13-27, 1992.

[3] J. Baranger and S. Wardi. Numerical analysis of a FEM for a transient viscoelastic flow. Comput. Methods. Appl. Mech. Engrg., 125:171-185, 1995.

[4] G. K. Batchelor. An Introduction to Fluid Dynamics. Cambridge University Press, Cambridge, 1967.

[5] R. B. Bird, R. C. Armstrong, and O. Hassager. Dynamics of Polymeric Liquids. John Wiley and Sons, Inc., 1987.

[6] J. U. Brackbill, D. B. Kothe, and C. Zemach. A continuum method for modeling surface tension. J. Comp. Phys., 100:335-354, 1992.

[7] S. C. Brenner and L. R. Scott. The Mathematical Theory of Finite Element Methods. SpringerVerlag Publishing, 1994.

[8] Y. Chang, T. Hou, B. Merriman, and S. Osher. A level set formulation of Eulerian interface capturing methods for incompressible fluid flows. J. Comp. Phys., 124:449-464, 1996.

[9] P. G. Ciarlet. The Finite Element Methods for Elliptic Problems. North-Holland Publishing, 1979 .

[10] Ph. Clément. Approximation by finite element functions using local regularization. RAIRO Anal. Numer., 2:77-84, 1975.

[11] V. J. Ervin and W. W. Miles. Approximation of time-dependent, viscoelastic fluid flow: SUPG approximation. SIAM J. Numer. Anal., 41(2):457-486, 2003.

[12] D. Gueyffier, J. Li, A. Nadim, R. Scardovelli, and S. Zaleski. Volume-of-fluid interface tracking with smoothed surface stress methods for three-dimensional flows. J. Comp. Phys., 152:423456, 1999.

[13] C. Guillope and J. C. Saut. Existence results for the flow of viscoelastic fluids with a differential constitutive law. Nonlinear Anal. Theory Methods Appl., 15:849-869, 1990.

[14] J. G. Heywood and R. Rannacher. Finite element approximation of the nonstationary NavierStokes problem. Part IV: Error analysis for second-order time discretization. SIAM J. Numer. Anal., 2:353-384, 1990.

[15] B. Liu. The analysis of a finite element method with streamline diffusion for the compressible Navier-Stokes equations. SIAM J. Numer. Anal., 1:1-16, 2000. 
[16] W. Miles. Modeling time-dependent, multicomponent, viscoelastic fluid flow. Ph.D. Theis, Clemson University, 2003.

[17] K. Najib and D. Sandri. On a decoupled algorithm for solving a finite element problem for the approximation of viscoelastic fluid flow. Numer. Math., 72:223-238, 1995.

[18] S. Osher and J. A. Sethian. Fronts propagating with curvature-dependent speed: Algorithms based on Hamilton-Jacobi formulations. J. Comp. Phys., 79:12-49, 1988.

[19] M. Renardy. Mathematical Analysis of Viscoelastic Flows. SIAM, Philadelphia, PA, 2000.

[20] D. Sandri. Finite element approximation of viscoelastic fluid flow: Existence of approximate solutions and error bounds. Continuous approximation of the stress. SIAM J. Numer. Anal., 31:362-377, 1994.

[21] M. Sussman, E. Fatemi, P. Smereka, and S. Osher. An improved level set method for incompressible two-phase flows. Comput. Fluids, 27(5-6):663-680, 1998.

[22] A. Tornberg and B. Engquist. A finite element based level-set method for multiphase flow applications. Comput. Visual. Sci., 3:93-101, 2000.

[23] J. E. Welch, F. H. Harlow, J. P. Shannon, and B. J. Daly. The MAC method. A computing technique for solving viscous, incompressible, transient fluid flow problems involving free surfaces. LASL Rep. LA-3425, 1965.

[24] D. F. Zhang and D. A. Zumbrunnen. Influences of fluidic interfaces on the formation of fine scale structures by chaotic mixing. Journal of Fluids Engineering, 118:40-47, 1996. 